\title{
Numerical study of the 3D failure envelope of a single pile in sand
}

\author{
Zheng $\mathrm{Li}^{\mathrm{a}, \mathrm{b}, *}$, Panagiotis Kotronis ${ }^{\mathrm{b}}$, Sandra Escoffier ${ }^{\mathrm{a}}$ \\ a LUNAM Université, IFSTTAR, GERS, SV, F-44341 Bouguenais, France \\ ${ }^{\mathrm{b}}$ LUNAM Université, Ecole Centrale de Nantes, Université de Nantes, CNRS, Institut de Recherche en Génie Civil et Mécanique (GeM), 1 Rue de la Noë, F-44321 Nantes, France
}

The paper presents a comprehensive study of the failure envelope (or capacity diagram) of a single elastic pile in sand. The behavior of a pile subjected to different load combinations is simulated using a large number of finite element numerical calculations. The sand is modeled using a constitutive law based on hypoplasticity. In order to find the failure envelope in the three-dimensional space (i.e. horizontal force $H$, bending moment $M$ and vertical force $V$ ), the radial displacement method and swipe tests are numerically performed. It is found that with increasing vertical load the horizontal bearing capacity of the pile decreases. Furthermore, the presence of bending moment on the pile head significantly influences the horizontal bearing capacity and the capacity diagram in the $H-M$ plane manifests an inclined elliptical shape. An analytical equation providing good agreement with the 3D numerical results is finally proposed. The formula is useful for design purposes and the development of simplified modeling numerical strategies such as macro-element.

\section{Introduction}

Pile foundations are widely used in geotechnical and offshore engineering. When subjected to a combination of horizontal $(H)$, vertical $(V)$ forces and bending moments $(M)$, a $3 \mathrm{D}(H-M-V)$ failure envelope (or bearing capacity diagram) is necessary in order to evaluate the safety of the pile-soil system. The first comprehensive experimental research studies on the bearing capacity of piles can be traced back to the 60s. Meyerhof [1] proposed a polar bearing capacity diagram adequate for a vertical pile. In 1972, Meyerhof and Ranjan [2] provided a new bearing capacity diagram taking into account the uplift mechanism based on the experimental results of Ranjan [3]. Meyerhof with his colleagues extended also the study of the vertical bearing capacity to batter piles [4], pile groups [5] and more recently to layered soils [6-8]. Their milestone studies mainly focused on the $H-V$ plane and provided valuable experimental data for the failure envelope of piles and pile foundation groups. There are also many experimental studies that have been carried out by many other researchers. Das et al. [9] performed model tests with short rigid piles embedded in loose sand. An empirical equation was proposed to consider the interaction

\footnotetext{
* Corresponding author at: LUNAM Université, IFSTTAR, GERS, SV, F-44341 Bouguenais, France. Tel.: +33 638804776

E-mail addresses: zheng.li@ec-nantes.fr (Z. Li), Panagiotis.Kotronis@ec-nantes.fr (P. Kotronis), sandra.escoffier@ifsttar.fr (S. Escoffier).
}

effect of combined loads. Patra and Pise [10] carried out model tests with slender aluminum piles in medium dense sand under oblique pull loads. They found the presence of horizontal load, if applied simultaneously, will significantly increase the ultimate vertical load. Smoothed pile surface had lager increase effect on the ultimate vertical load.

From the numerical point of view, Cho [11] studied the vertical and horizontal bearing capacities of suction piles and proposed a piecewise empirical equation. Achmus and Thieken [12] focused on the bearing behavior under vertical and horizontal loads and the effects of load interactions on the pile-soil stiffness reduction. Mroueh and Shahrour [13] looked at the bearing capacity of a single vertical pile comparing the results with that of a single inclined pile and Fan and Meng [14] performed numerical swipe tests to investigate the failure envelope of a pipe pile foundation in the $H-M-V$ 3D space. In 2011, Correia [15] derived the failure envelope in $H-M$ plane for single vertical pile embedded in clay by a semi-analytical approach.

In this paper, the failure envelope of a single elastic vertical pile in sand is numerically investigated via swipe tests and a large number of radial displacement controlled tests. A complete 3D failure envelope in $H-M-V$ space is provided. The analytical equation proposed by Meyerhof and Ranjan [2] is validated and adopted in the $H-V$ plane. For the $H-M-V$ space however, a new 3D analytical failure envelope is introduced. The proposed failure envelope is useful for the development of new simplified modeling strategies 
for soil-structure interaction problems (e.g. macro-elements [16-18]) that can be applied in design engineering offices [19].

\section{Numerical model}

\subsection{Soil constitutive law}

The performance of a soil-pile interaction numerical strategy greatly depends on the adopted soil constitutive law. The Mohr-Coulomb [20] and the Drucker and Prager [21] elasto-plastic laws are the most commonly used constitutive relations in geotechnical engineering. In this paper however, the choice is made to use a simple hypoplastic constitutive law. The term hypoplasticity was introduced by Dafalias [22] to designate a particular type of bounding surface plasticity with a vanishing elastic domain [23]. In comparison with elastoplastic models hypoplasticity can provide a more smooth numerical response [23]. An outline of the hypoplasticity theory was presented by Kolymbas in 1991 [24].

In the following, a constitutive law similar to an elastic perfectly plastic Drucker-Prager model is formulated according to the hypoplasticity theory and it is used to numerically reproduce the behavior of a single pile in sand. The basic framework of the hypoplastic formulation is provided with the following equation [25, 26] (bold letters define hereafter tensors and vectors, the dot ".." symbol is the derivative with respect to time and \|\| the norm of a tensor):

$$
\dot{\mathbf{T}}=\mathcal{L} \mathbf{D}+\mathbf{N}\|\mathbf{D}\|
$$

where $\mathbf{T}$ and $\mathbf{D}$ are the stress rate and stretching rate tensors. In contrast with elasto-plasticity, in hypoplasticity the tangent stiffness matrix varies continuously with the direction of the velocity, a property known as incremental nonlinearity $[27,28]$. The nonlinear behavior of the material results thus from the interaction between the stiffness matrix $\mathcal{L}$ and vector $\mathbf{N}$.

It is considered hereafter that stiffness matrix $\mathcal{L}$ depends on the bulk modulus $K$ and the shear modulus in the elastic range $G_{\max }$. It has the following form (the Lamé coefficient $\mu=G_{\max }$ according to Hooke's law):

$$
\mathcal{L}=\left[\begin{array}{cccccc}
K+4 \mu / 3 & K-2 \mu / 3 & K-2 \mu / 3 & 0 & 0 & 0 \\
K-2 \mu / 3 & K+4 \mu / 3 & K-2 \mu / 3 & 0 & 0 & 0 \\
K-2 \mu / 3 & K-2 \mu / 3 & K+4 \mu / 3 & 0 & 0 & 0 \\
0 & 0 & 0 & \mu & 0 & 0 \\
0 & 0 & 0 & 0 & \mu & 0 \\
0 & 0 & 0 & 0 & 0 & \mu
\end{array}\right]
$$

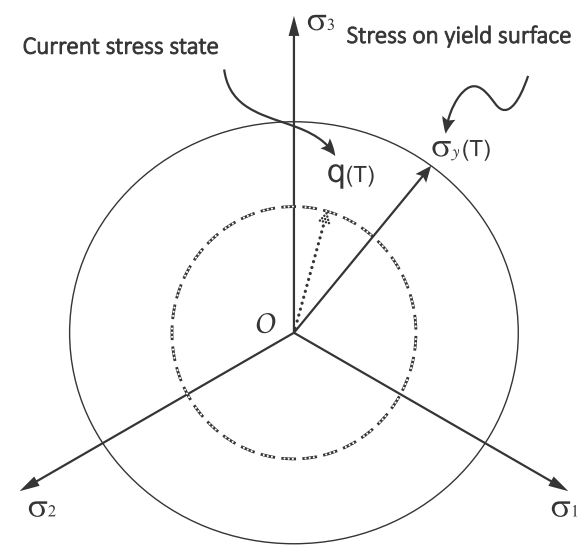

(a)
For a frictional material like sand, $G_{\max }$ is influenced by the mean effective confining pressure $p$ and the void ratio $e$ [29-32]:

$G_{\max }=f_{n}(e, p)$

It is usually considered that

$G_{\max }=A \frac{(B-e)^{2}}{(1+e)} p^{C}$

where $A, B, C$ are material constants.

The constitutive tensor $\mathbf{N}$ is defined following the approach proposed by Niemunis [26]:

$\mathbf{N}(\mathbf{T})=-y(\mathbf{T}) \mathcal{L} \mathbf{m}(\mathbf{T})$

where $y(\mathbf{T})$ is a scalar function, named degree of nonlinearity, that controls the variation of the nonlinear term and $\mathbf{m}(\mathbf{T})$ defines the plastic flow direction. In this paper, the scalar function $0 \leqslant y(\mathbf{T}) \leqslant 1$ is chosen as a function of the current stress $q(\mathbf{T})=\sqrt{3 J_{2}}$ and a predefined limit stress $\sigma_{\mathrm{y}}(\mathbf{T})$ as follows:

$y(\mathbf{T})=\left(\frac{q(\mathbf{T})}{\sigma_{\mathrm{y}}(\mathbf{T})}\right)^{n_{c}}$

with $n_{c}$ is a constant that controls the isotropic evolution of $y(\mathbf{T})$ (the influence of $n_{c}$ will be discussed hereafter) and $J_{2}$ the second invariant of the deviatoric stress tensor. The ratio $q(\mathbf{T}) / \sigma_{\mathrm{y}}(\mathbf{T})$ measures the distance between the current stress surface (which expands isotropically) and the predefined yield surface, see Fig. 1(a). It is considered hereafter that the predefined yield stress $\sigma_{y}(\mathbf{T})$ cannot be surpassed, meaning that once the yield surface reached $q(\mathbf{T})-\sigma_{y}(\mathbf{T})=0$.

The objective is to use an hypoplastic constitutive law similar to the elastic perfectly plastic Drucker-Prager model, the chosen $\sigma_{y}(\mathbf{T})$ has to lie on the Drucker-Prager yield surface (or on compression corners of the Mohr-Coulomb criterion [33,34], see Fig. 1(b)) depending on the mean effective confining pressure $p(\mathbf{T})$ and the material frictional angle $\phi$. The way to do this is explained hereafter. form:

The Drucker Prager yield criterion has the following general

$F=q(\mathbf{T})-M_{c} p(\mathbf{T})-k_{c}=0$

where $M_{c}$ and $k_{c}$ are material constants expressed in terms of the friction angle $\phi$ and the cohesive strength $c$ :

$M_{c}=\frac{6 \sin \phi}{3-\sin \phi}$

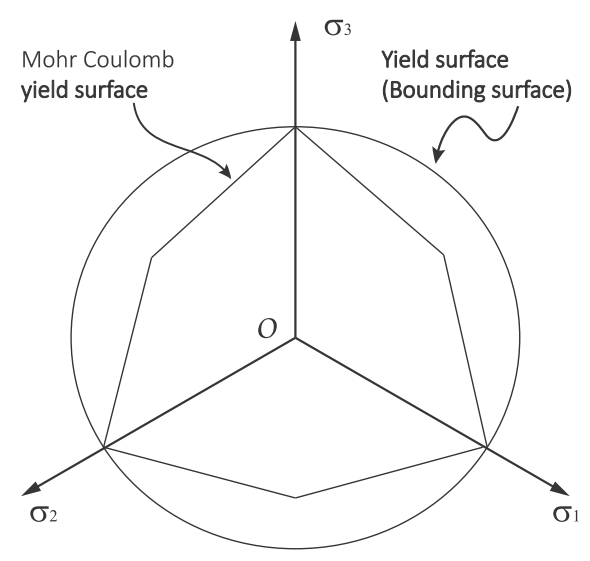

(b)

Fig. 1. (a) Cross-sections of the current stress and yield surfaces and (b) matching the predefined yield surface with the Mohr-Coulomb criterion. 


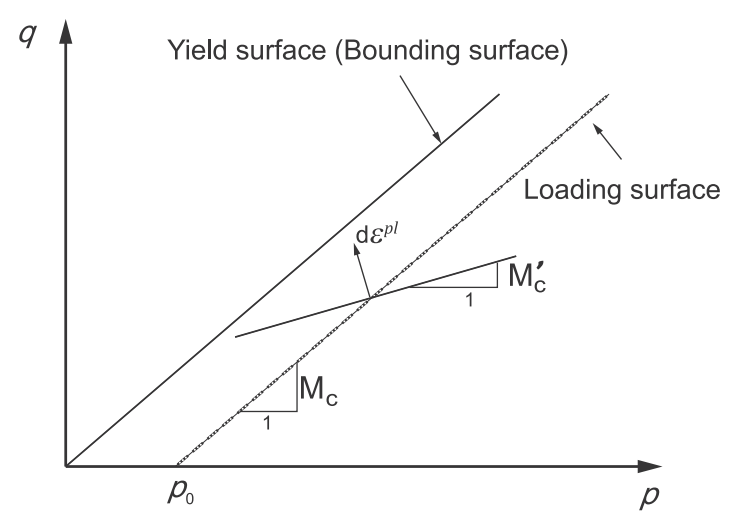

Fig. 2. Plastic flow direction.

$k_{c}=6 c \frac{\cos \phi}{3-\sin \phi}$

Assuming a dry sand and thus a zero cohesive strength $(c=0)$, $k_{c}=0$ and the yield function reads:

$F=q(\mathbf{T})-M_{c} p(\mathbf{T})=0$

From Eq. (10) it is obvious that the constant parameter $M_{c}$ defines the slope of the yield surface (see Fig. 2). According to Eq. (10) it can also be assumed that $\sigma_{y}(\mathbf{T})=M_{c} p(\mathbf{T})$ and thus the scalar function of Eq. (6) reads:

$y(\mathbf{T})=\left(\frac{q(\mathbf{T})}{\sigma_{\mathrm{y}}(\mathbf{T})}\right)^{n_{c}}=\left(\frac{q(\mathbf{T})}{M_{c} p(\mathbf{T})}\right)^{n_{c}}$

The plastic flow direction $\mathbf{m}(\mathbf{T})$ is defined according to the bounding surface model $[22,35,36]$. The current stress $q(\mathbf{T})$ is assumed to lie on a loading surface $f$ which has a similar shape with the yield surface $F$ (bounding surface), see Fig. 2. The expression of the loading surface can be thus defined as:

$f=q(\mathbf{T})-M_{c}\left(p(\mathbf{T})-p_{0}\right)=0$

with

$p_{0}=\frac{1}{M_{c}}\left(\sigma_{y}(\mathbf{T})-q(\mathbf{T})\right)=\frac{1}{M_{c}}\left(M_{c} p(\mathbf{T})-q(\mathbf{T})\right)$

When $q(\mathbf{T})$ reaches the limit stress $\sigma_{y}(\mathbf{T})=M_{c} p(\mathbf{T}), p_{0}=0$ and the loading surface coincides with the bounding surface. Due to the similar shapes of the yield and loading surfaces, for given stress state $(p(\mathbf{T}), q(\mathbf{T}))$, the directions normal to the two surfaces are identical. In order to determine the direction of the plastic strain increment $d \varepsilon^{p l}$ (see Fig. 2), one can thus only refer to the yield surface. As usually done for soils, a non-associated flow rule is chosen:

$G=q(\mathbf{T})-M_{c}^{\prime} p(\mathbf{T})$

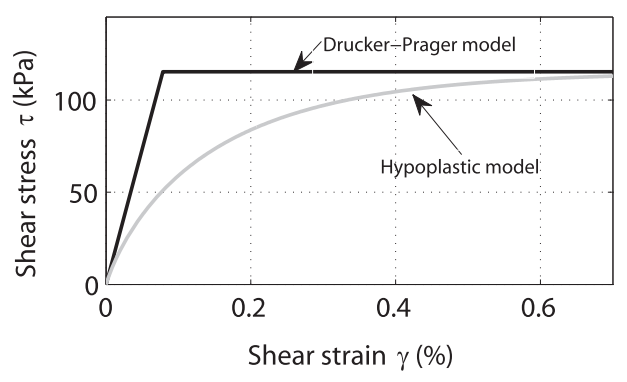

(a) with

$M_{c}^{\prime}=\frac{6 \sin \psi}{3-\sin \psi}$

and $\psi$ the sand dilation angle. The parameter $M_{c}^{\prime}$ defines the direction of the plastic strain increment (see Fig. 2). The plastic flow direction is given by Eq. (14):

$\mathbf{m}(\mathbf{T})=\frac{\partial G / \partial \mathbf{T}}{\|\partial G / \partial \mathbf{T}\|}$

The comparison of the results on a simple shear test using the Drucker-Prager model and the proposed hypoplastic model is shown in Fig. 3(a). In this example, the friction and dilation angles of the sand are assumed equal to $33^{\circ}$ and $0^{\circ}$ respectively and the mean confining pressure $149 \mathrm{kPa}$. The hypoplastic model provides the same ultimate strength as the Drucker-Prager model but follows a more smooth transition curve. Finally, the influence of the coefficient $n_{c}$ on the response of model is shown in Fig. 3(b).

\subsection{Calibration and validation of the soil constitutive law for Fontainebleau sand}

In this section, the soil constitutive law is calibrated and validated using experimental data on dry Fontainebleau sand. The homogeneous sand has a mid-particle diameter $D_{50}$ around $0.206 \mathrm{~mm}$, a density, $\rho_{s}=2.65 \mathrm{~g} / \mathrm{cm}^{3}$ and a minimum and maximum void ratio $e_{\min }=0.510$ and $e_{\max }=0.882$, respectively [37]. The main parameters of the soil model are summarized in Table 1.

The elasticity constants $A, B, C$ and the void ratio $e$ are used to define the elastic behavior of the soil. According to Eqs. (3) and (4), the initial shear modulus $G_{\max }$ (in MPa) depends on the mean confining pressure $p$ (in $\mathrm{kPa}$ ) and the void ratio $e$. Delfosse-Ribay et al. [38] proposed the following values for the Fontainebleau sand used in dynamic centrifuge tests: $A=200, B=2.17$ and $C=0.47$. The Young's modulus $E$ is then calculated as:

$E=2 G_{\max }(1+v)$

where $v=0.25$ is the Poisson's ratio.

In order to properly identify the parameter $n_{c}$ of the hypoplastic law, experimental results from dynamic centrifuge tests with the Fontainebleau sand under free-field conditions are used [39]. Free-field tests were conducted with an Equivalent Shear Beam

Table 1

Model parameters used in the simulation of drained triaxial tests.

\begin{tabular}{llllllll}
\hline & $A$ & $B$ & $C$ & $e$ & $n_{c}$ & $\phi_{c}\left({ }^{\circ}\right)$ & $\psi\left({ }^{\circ}\right)$ \\
\hline Dense sand & 200 & 2.17 & 0.47 & 0.577 & 0.4 & 33 & 9 \\
Loose sand & 200 & 2.17 & 0.47 & 0.707 & 0.4 & 33 & 3 \\
\hline
\end{tabular}

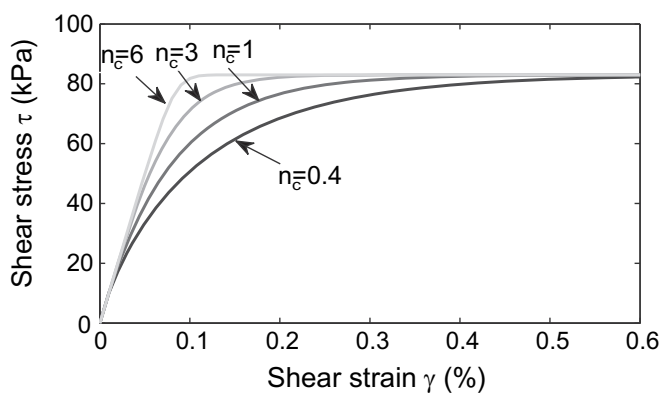

(b)

Fig. 3. Comparison of the Drucker-Prager model with the proposed hypoplastic model. 
(ESB) container [40,41] at IFSTTAR Nantes that allows shear deformation and shear wave propagation in the soil medium. The stress-strain behavior and the shear modulus degradation curve were derived following a post-processing strategy based on the measurements provided by a dense network of accelerometers. Details about the procedure can be found in [39] (see also [42]). By fitting the shear modulus degradation curve with the experimental data (see Fig. 4), $n_{c}$ is found to be approximately equal to 0.4 .

The friction angle $\phi$ and the dilation angle $\psi$ are required to reproduce the strength and volumetric change of the soil. In [43], the peak friction angle and the critical state friction angle of Fontainebleau sand are given equal to $\phi_{\text {peak }}=41.8^{\circ}$ and $\phi_{c}=33^{\circ}$ respectively. In the following, the soil model is validated using drained triaxial test data where the critical friction angle is adopted to reproduce the soil strength and different dilation angles are used for the case of dense and loose sand.

The results of the triaxial tests for dense and loose sand are shown in Figs. 5 and 6. Different confining pressures are considered (50 kPa, $100 \mathrm{kPa}$ and $200 \mathrm{kPa}$ ). As expected for the dense sand (see Fig. 5(a) and (b)), the hypoplastic model cannot capture the peak state. Nevertheless, using the critical state friction angle the ultimate strength of the soil is well reproduced. In order to fit the tendency of the volumetric changes the dilation angle is estimated to be $\psi=9^{\circ}$.

For the case of loose sand (see Fig. 6(a) and (b)), using the critical state friction angle and a lower dilation angle $\psi=3^{\circ}$ the

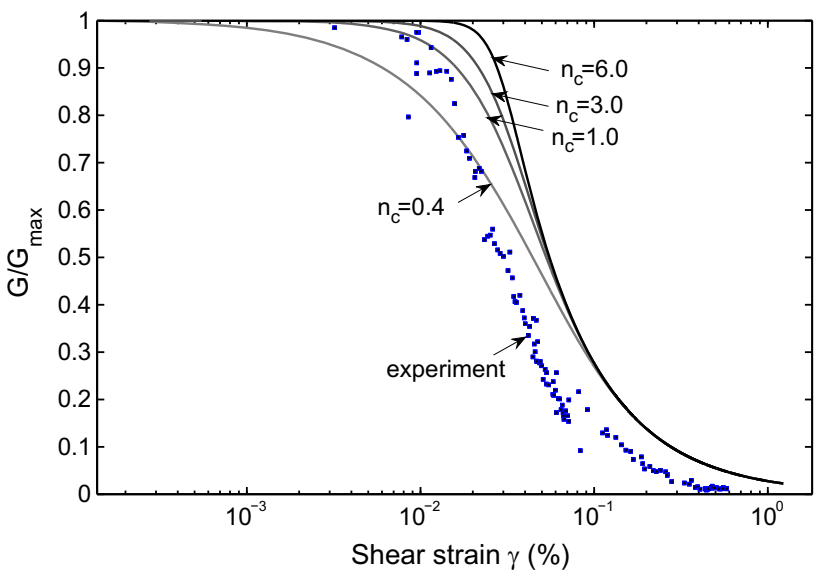

Fig. 4. Comparison of the numerical $G / G_{\max }$ curve with the experimental data (after Li et al. [39]).

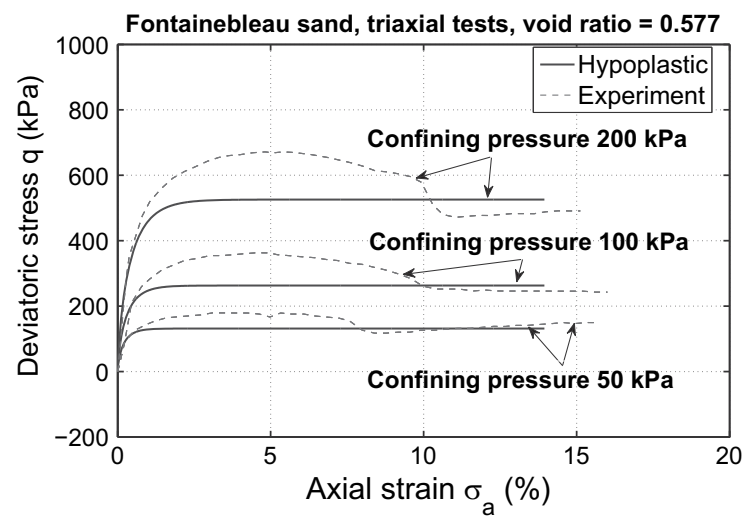

(a) proposed hypoplastic model captures approximately both the stress-strain and the volumetric change behavior.

Although the model cannot capture the peak state for the case of dense sand, it is assumed in the following that the soil in the vicinity of the pile undergoes large deformations and reaches the ultimate critical state (the purpose of the paper is to quantify the ultimate failure envelope of the soil-pile system). By using the critical state friction angle, the residual strength of the soil is well captured. One has to keep in mind however that the proposed hypoplastic model does not use the concept of critical state and cannot produce a constant volumetric strain with the critical friction angle.

\subsection{Finite element mesh and boundary conditions}

A numerical model of a single vertical pile in soil is carried out using the finite element code ABAQUS standard [44]. Taking advantages of the symmetry of the problem, only half of the soil-pile system is modeled (see Fig. 7, $D$ is the diameter of the pile). Both sand and pile are reproduced using 3D solid elements (the total number of elements used for soil is 14958). The pile is assumed to have a linear elastic behavior while the sand follows the simple hypoplastic model presented in Section 2.1. The behavior at the interface between the pile and the soil is modeled using a classical Coulomb model where the tangential frictional stress is proportional to the normal stress. The contact interface can open and close. Due to the lack of experimental data to quantify the limit stress in the tangential direction, no threshold $\left(\tau_{\max }\right)$ on the tangential frictional stress is adopted. The contact behavior between the pile and the soil is considered using a friction coefficient $\mu=\tan \left(\phi_{c}\right)=0.65$ related to the critical friction angle $\phi_{c}=33^{\circ}$ of the soil. For the contact behavior, a penalty algorithm is adopted [44]. The objective of the numerical study is to capture the 3D failure envelope, large deformations and geometric non-linearity are considered in the simulations. Furthermore, an adaptive re-meshing technique, Arbitrary Lagrangian Eulerian (ALE), is necessary [44]. Otherwise, because of numerical convergence problems due to severe distortions of the finite elements the code stops before reaching the material failure conditions.

Nodal displacements are fixed in the $X, Y$ and $Z$ directions at the base of the finite element mesh. The displacements at the other lateral boundaries are blocked in the normal directions (see Fig. 7). There is no load eccentricity on the pile head i.e. the pile head is at the ground surface level.

A mesh sensitivity study, adopting the $H$-convergence method, is carried out to ensure that the number of finite elements is sufficient to provide accurate results. Three finite element meshes are studied hereafter: a coarse mesh (with 6116 elements, Fig. 8(a)),

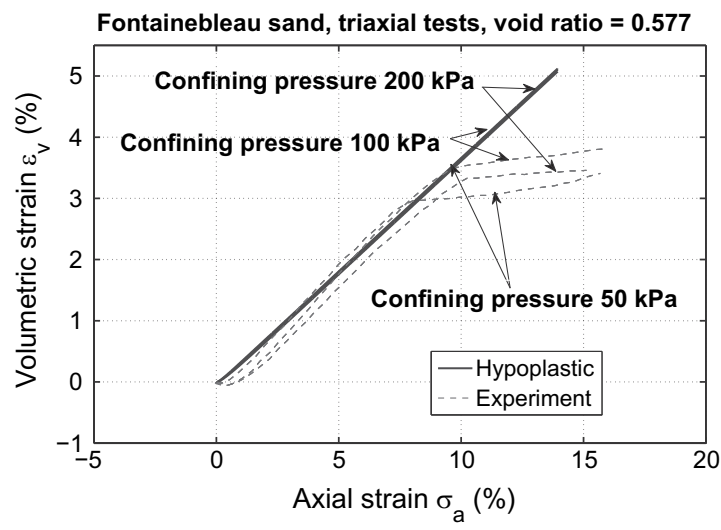

(b)

Fig. 5. Triaxial compression tests on dense Fontainebleau sand, void ratio $=0.577$, simulation vs. experiments 


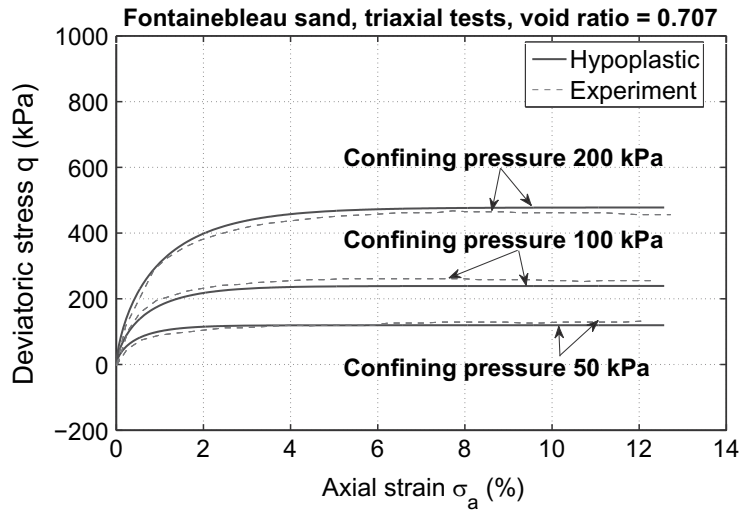

(a)

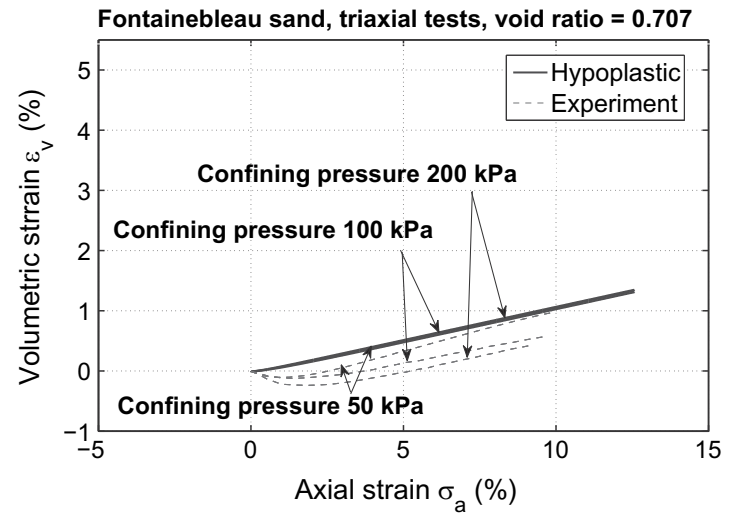

(b)

Fig. 6. Triaxial compression tests on loose Fontainebleau sand, void ratio $=0.707$, simulation vs. experiments.

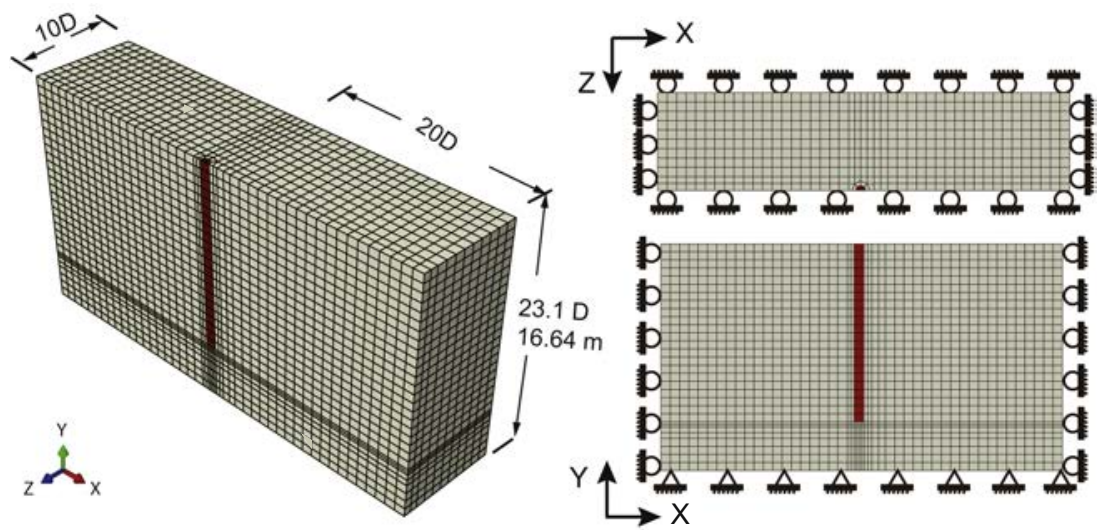

Fig. 7. Finite element model for a single vertical pile in soil.

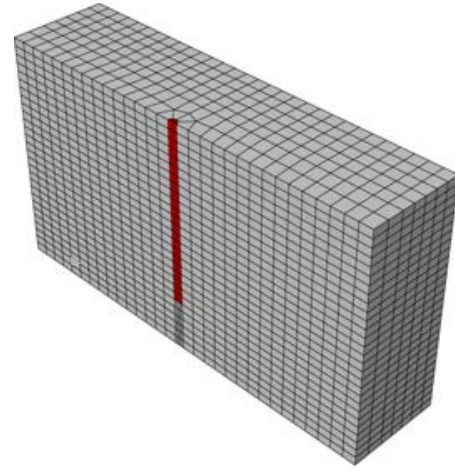

(a)

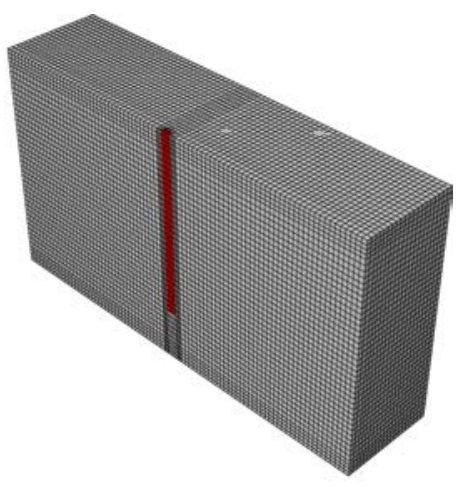

(b)

Fig. 8. (a) Coarse mesh and (b) fine mesh.

the current mesh (with 14,958 elements, Fig. 7 and a fine mesh (66,238 elements, Fig. 8(b)). Dense sand is assumed with the parameters mentioned in Table 1 . The pile section stiffness is taken equal to $E_{p} I_{p}=2500 \times 10^{6} \mathrm{~N} \mathrm{~m}^{2}$. Comparison of the results is shown in Fig. 9. Increasing the number of elements improves the accuracy of the finite element computations. However, the difference between the fine and the current meshes results is of the order of $5 \%$. The fine mesh is computationally very expensive, the current mesh discretization is adopted for the rest of the paper.

\section{Validation of the numerical model}

In order to validate the proposed finite element model, results from two centrifuge pile test campaigns are used hereafter. The first series of tests were performed by Rosquoët [45] to study the lateral resistance and the second by Guefrech et al. [46] to investigate the bearing capacity of a vertical pile embedded in Fontainebleau sand. In both campaigns, the tested pile head was above the ground surface. In the following, this load eccentricity 


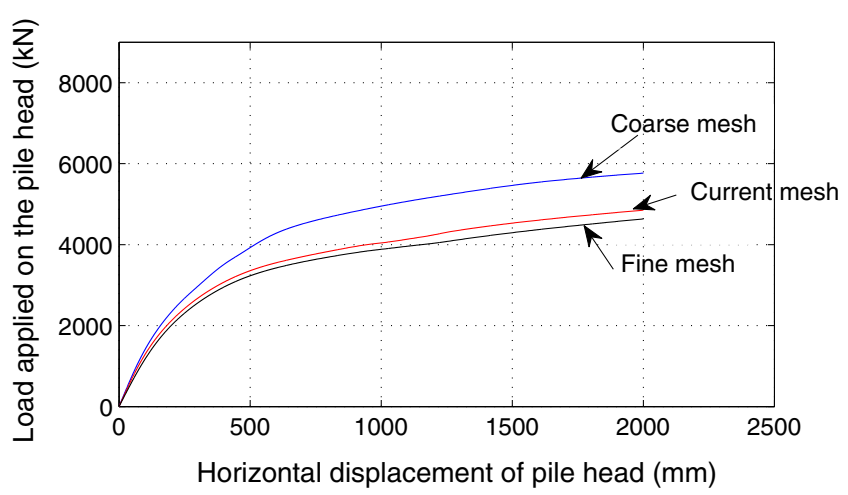

Fig. 9. Mesh sensitivity study.

is introduced in the finite element model by increasing the actual length of the pile above the soil surface. Both the two centrifuge tests concern dense sand and the ultimate strength of the soil-pile system, critical friction angle of the sand is used. All the parameters are given hereafter in the prototype (real) scale.

Rosquoët [45] applied monotonically increasing horizontal force on a single vertical pile embedded in Fontainebleau sand submitted to a centrifuge gravity level of $40 \mathrm{~g}$ ( $\mathrm{g}$ is the gravity acceleration). The diameter of the pile was $0.72 \mathrm{~m}$ and its slenderness (length/diameter) ratio is 15 .

The main parameters adopted in the finite element model to reproduce Rosquoët's centrifuge tests are summarized in the first row of Table 1 (dense sand). In order to be consistent with the centrifuge test, $e=0.58$ is used. The pile is modeled using a linear elastic constitutive law and has a section stiffness of $E_{p} I_{p}=2638 \times 10^{6} \mathrm{~N} \mathrm{~m}^{2}$ (with $E_{p}$ the Young's modulus and $I_{p}$ the moment of inertia of the pile). The diameter of the pile is $0.72 \mathrm{~m}$. A monotonically increasing horizontal displacement is applied on the pile head, see in Fig. 10(a). The comparison of the numerical results with the experimental data in Fig. 10(b) shows that the numerical model reproduces correctly not only the evolution of the lateral strength of the vertical pile but also its ultimate value.

Guefrech et al. [46] studied the bearing capacity of a vertical pile embedded in Fontainebleau Sand under a centrifuge gravity level of $23 \mathrm{~g}$. A monotonically increasing vertical force was applied on the pile head. The sand relative density was around $D_{r}=86 \%$, the diameter of the pile was $0.4 \mathrm{~m}$ and its slenderness (length/ diameter) ratio 30 . The surface of the pile is rough. The values of the main parameters adopted in the finite element model are listed in the first row of Table 1 (dense sand). In order to be consistent with the centrifuge test, $e=0.56$ is used.
The pile is again modeled using a linear elastic constitutive law and has a section stiffness of $E_{p} I_{p}=100 \times 10^{6} \mathrm{~N} \mathrm{~m}$. A monotonically increasing vertical displacement is applied on the pile head, see in Fig. 11(a). Numerical results are compared with the experimental results in Fig. 11(b). The performance of the model is satisfactory. It can be noticed however that the model works better for the case of loose sand that for dense sand, where it misses its peak strength. Nevertheless, in both cases the model reproduces correctly the ultimate failure envelope of a single pile in sand (see also Section 2.2).

\section{Numerical study of the 3D failure envelope}

\subsection{Parameters of the finite element model}

The finite element model, validated in Section 3 with the results of two centrifuge experimental campaigns, is used hereafter to study the 3D failure envelope of a single pile embedded in Fontainebleau sand. The pile has a length of $13 \mathrm{~m}$, a diameter of $0.72 \mathrm{~m}$, a slenderness ratio of 18 and the pile head is considered on the ground surface level. A linear elastic constitutive law is adopted for the pile while the hypoplastic law presented in Section 2 is considered for dense Fontainebleau sand. The parameters of the hypoplastic law are listed in the first row of Table 1 (dense sand). Poulos and Davis [47] proposed a method to classify piles in different categories based on the flexibility factor $K_{r}$, see Eq. (16):

$K_{r} \geqslant 0.1$ : rigid piles.

$0.01 \leqslant K_{r}<0.1$ : relative stiff piles.

$0.001 \leqslant K_{r}<0.01$ : medium flexible piles.

$1 \times 10^{-4} \leqslant K_{r}<0.001$ : flexible piles.

$1 \times 10^{-5} \leqslant K_{r}<1 \times 10^{-4}$ : very flexible piles.

where $K_{r}$ is defined as:

$K_{r}=\frac{E_{p} I_{p}}{E_{s} L_{p}^{4}}$

with $E_{p} I_{p}$ the section stiffness of the pile, $L_{p}$ its embedded length and $E_{s}$ the soil secant modulus (or deformation modulus). In the following, $E_{p} I_{p}=5.05 \times 10^{8} \mathrm{~N} \mathrm{~m}^{2}$ and $L_{p}=13 \mathrm{~m}$. To determine the soil deformation moduli $E_{s}$, several experimental methods were proposed by Poulos and Davis [47]. In this paper, another method is adopted. Jardine et al. [48] and Mair [49] have shown that the typical shear strain level around piles falls in the range $0.01 \sim 0.1 \%$. With the $G / G_{\max }$ curve, the range of the secant shear modulus $G_{s}$ can thus be determined and then converted to $E_{s}$. In the following, the $G / G_{\max }$ curve proposed by Li et al. [39] for a Fontainebleau sand is adopted and $G_{\max }$ is calculated using Eq. (4). It is found that the

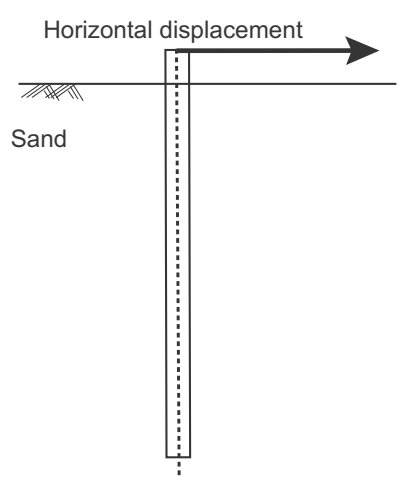

(a)

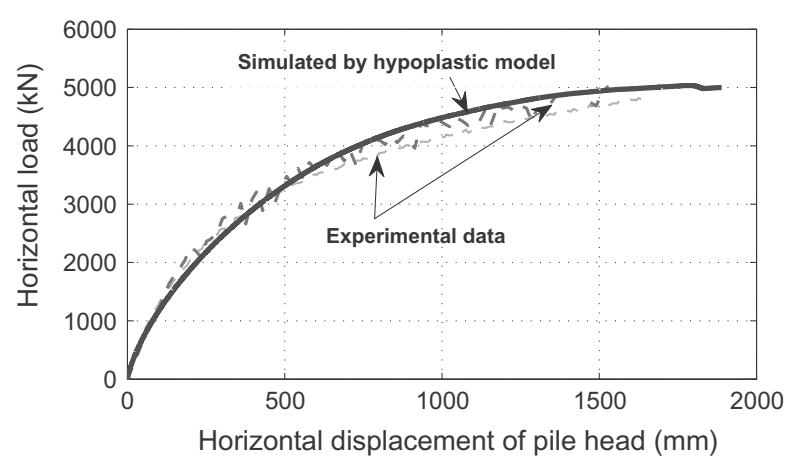

(b)

Fig. 10. (a) Schematic of the loading configuration and (b) numerical (hypoplastic model) vs. experimental data - Rosquoët's centrifuge tests (lateral loading) [45]. 


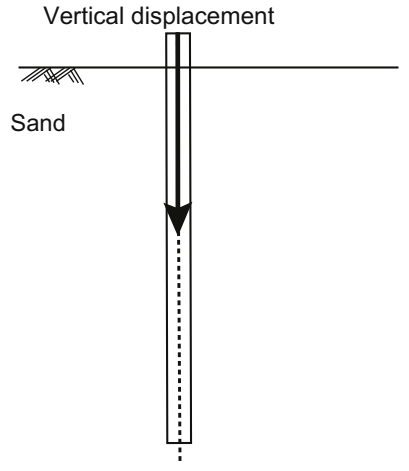

(a)

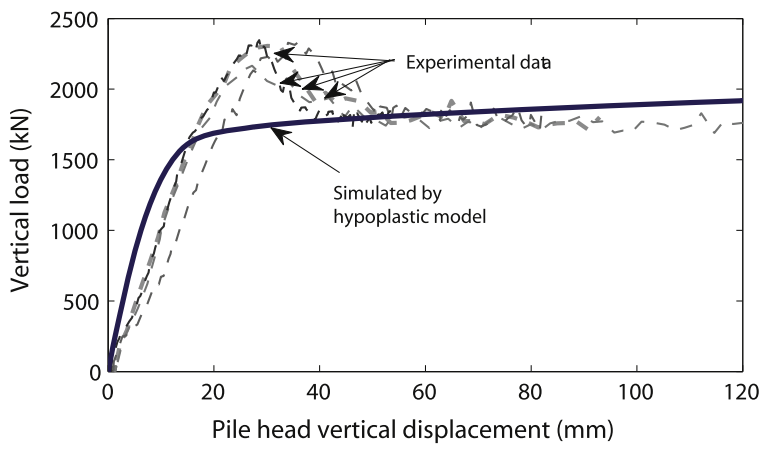

(b)

Fig. 11. Numerical (hypoplastic model) vs. experimental data, Guefrech et al. centrifuge tests (vertical loading) [46].

average secant modulus $E_{s}$ is in the range $67.6 \sim 176.8 \mathrm{MPa}$. Therefore, the flexibility factor falls in the range $1.0 \times 10^{-4} \sim 2.6 \times 10^{-4}$ and the pile in the FEM model can be classified as flexible. Following also the method proposed by Santos and Correia [50] (reference strain $\left.\gamma_{0.7}\right) K_{r}$ is estimated $2.5 \times 10^{-4}$, which also indicates a flexible behavior of the pile.

\subsection{D numerical failure envelope}

In order to numerically reproduce the $3 \mathrm{D}$ failure envelope, the procedure proposed by Gottardi et al. [51] is adopted. The authors applied two different loading paths on circular footings experimentally:

- Swipe tests: a vertical displacement is at first applied on the foundation until the vertical force reaches the desired level. Then, an increasing horizontal displacement is imposed on the foundation. Swipe analyses is introduced by Tan [52] and has been frequently adopted in several experimental [53] and numerical [54-56] studies.

- Radial displacement tests: tests where horizontal, vertical and/or rotational displacements are applied simultaneously while the ratio between the applied displacements (or combined rotation-displacement increments) is kept constant.

In this paper, the numerical radial displacement test is followed as the main numerical approach. The sign conventions for the pile head loads (horizontal force, vertical force and bending moment) are presented in Fig. 12. For the decomposed forces, vertical component acting downwards as a compression force is considered with a positive sign; horizontal component acting on the left is considered positive,vice versa. For the bending moment, the moment acting anti-clockwise is positive, vice versa.

\subsubsection{Numerical failure envelope in the $H-V$ plane}

To investigate the form of the failure surface in the $H-V$ plane, free pile head conditions $(M=0)$ are considered. As shown in Fig. 13, a displacement is applied on the top of the pile head (that can rotate freely) in a certain direction $\delta$. The angle of the displacement $\delta$ varies from $0 \sim 360^{\circ}$ to scan the failure surface in all directions. The value of the displacement is important to ensure that we reach the ultimate strength.

Different methods can be found in the literature to determine the pile capacity. The tangent intersection method proposed by Butler and Hoy [57], the Davisson's limit offset method [58] or $[59,60]$ are used for the vertical bending capacity. For the horizontal bearing capacity, the asymptote-tangent method is often

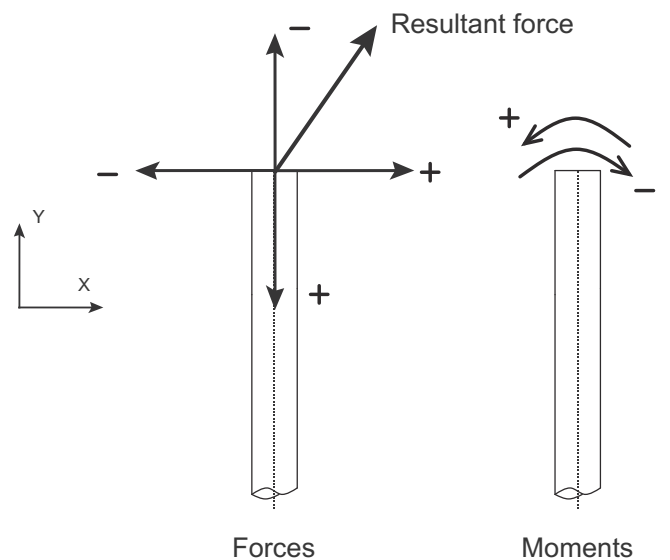

Fig. 12. Sign conventions for the pile head loadings.

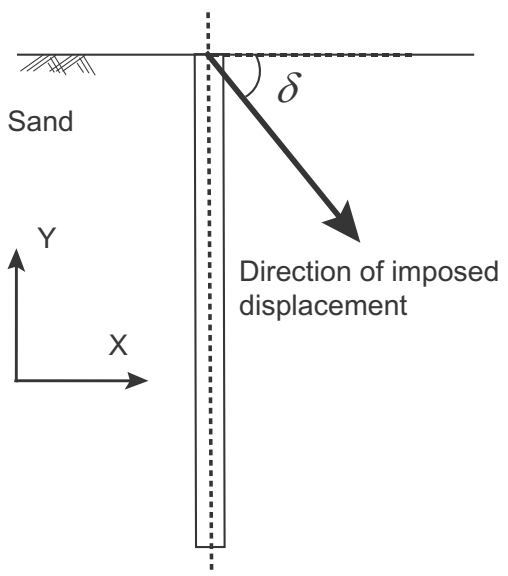

Fig. 13. Radial displacements tests in the $H-V$ plane.

adopted $[61,62]$. The above-mentioned methods are pile deflection based.

In this work, the final strength is chosen as the point where numerical calculation diverges. More specifically, large displacements are applied on the pile head. The finite element code stops (converge problems) when the bearing capacity is reached. The objective of the numerical study is to capture the 3D failure envelope surface, large deformations are considered and a re-meshing technique is necessary (ALE). By connecting the values at the ends of the different load paths (see the round points at Fig. 14), the 


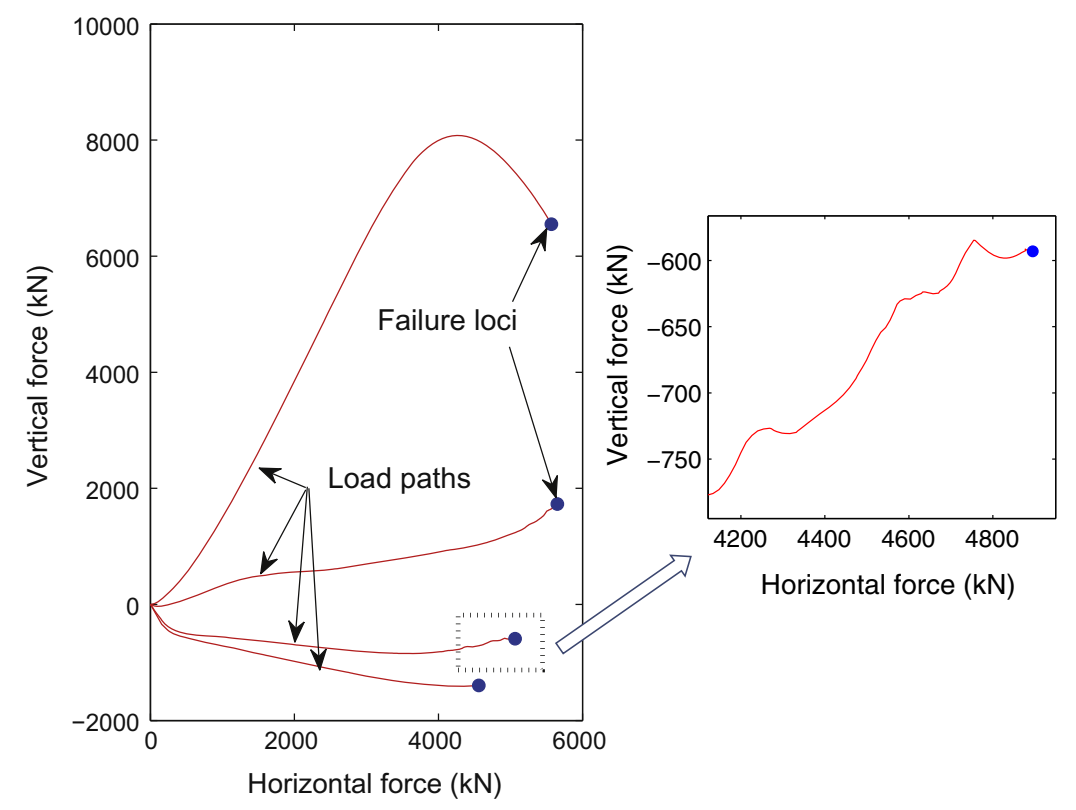

Fig. 14. Determination of the failure loci.

complete failure surface is thus obtained. It can however be observed in Fig. 14 that due to numerical convergence difficulties (the global stiffness is highly ill-conditioned) when the load paths approach the end points, jumps can appear and the paths are not so smooth. Examples of load paths in the $H-V$ plane from the numerical radial displacement tests are shown in Fig. 15(a).

Numerical swipe tests are also performed in $H-V$ plane and the results are plotted in Fig. 15(b). It can be seen that the load is first increased in the vertical axis up to a certain level. Then, a horizontal displacement is applied and the forces follow a path until the ultimate strength is reached. In this way, the swipe tests follow approximately the surface of the failure envelope [51]. The arrows in Fig. 15(b) denote the directions of the corresponding loading paths. Another two numerical swipe tests with more complex load paths were carried out, the horizontal displacement varies in a cyclic way in order to investigate the stress boundary in $H-V$ plane, the result is shown in Fig. 15(c), the solid line and the dash-line are different load paths. The numerical results from both the radial displacement tests and swipe tests provide similar failure envelopes. The failure envelope thus could be regarded as load path independent and has a fixed shape and position in the $H-V$ space. In the following, the radial displacement method is preferentially chosen to derive the failure envelope.

A large number (around 500) of numerical radial displacement tests are performed and the ultimate strength (or failure locus) of each test is plotted in Fig. 15(d). The data points form an unsmoothed curve, due to the high non-linearity: for the simulations in $H-V$ plane, the angles ( $\delta$, in Fig. 13) of the displacements are evenly varied; the obtained stress paths however are curved (see Figs. 14 and 15(a)). This is the reason why the data points in the $H-V$ force space are unevenly distributed. The main results are summarized hereafter:

1. The cross-section of the failure envelope is (almost) symmetric about the vertical force axis but not about the horizontal force axis.

2. A single vertical pile has a larger bearing capacity in compression than in tension.

3. With the increase of the vertical load (compression or tension), strong interaction between the vertical and horizontal loads is observed and the horizontal bearing capacity decreases.
The main reason behind the third remark is probably related to the plastic zone formed in the vicinity of the pile. In a classical elasto-plastic constitutive law, total strains are divided into an elastic and plastic part. The hypoplastic model however does not distinguish elastic from plastic strains and therefore the plastic zone cannot be accurately represented by the accumulated plastic strain. In the following, the plastic zone is represented using the nonlinear factor of the hypoplastic constitutive model, see Eq. (6). The range of this nonlinear factor is $[0,1]$. When the factor is close to 1 , the soil is highly nonlinear; when it is close to 0 , the soil presents an (approximately) elastic linear behavior. The nonlinear factor can be plotted as a state variable in Abaqus [44]. Fig. 16 shows the results of three loading cases: (a) pure horizontal loading, (b) pure vertical loading and (c) inclined loading, (red color denoting very important nonlinearity). For the pure horizontal loading, Fig. 16(a) shows that plasticity develops on both sides of the pile while (almost) no plasticity appears in the zone closed to the pile tip. For the pure vertical loading, Fig. 16(b), plasticity develops near the pile tip and both pile sides near the pile tip. Fig. 16(a) and (b) are characteristic of the two extreme loading cases. For the inclined loading case, Fig. 16(c), it seems that the presence of nonlinearity near the pile tip mitigates the nonlinearity on the pile sides and therefore reduces the horizontal bearing capacity. With increasing nonlinearity at the pile tip the horizontal resistance of the pile is expected to reduce.

The obtained failure envelope is similar to the experimental data from Meyerhof and Ranjan [2] corresponding to a single vertical pile in dense sand with a length to diameter ratio $L / D$ equal to 15. The authors indicated that the ultimate resultant load for vertical piles in dense sand decreases when the resultant force varies from a standalone vertical load to a standalone horizontal load (i.e. the horizontal component of the resultant force decreases with increasing vertical load). According to the results shown in Fig. 15(d), when $V=0$ the horizontal bearing capacity $H_{0}$ is estimated around $5000 \mathrm{kN}$; when the vertical load increases at $V=20,000 \mathrm{kN}$ (i.e. $80 \%$ of the ultimate vertical bearing capacity), the horizontal bearing capacity is estimated around $2700 \mathrm{kN}$ (considering the average value of the scatter data points). The horizontal bearing capacity is thus found reduced by $48 \%$.

Remark: It should be notice that according to the Abaqus 6.10 manual [44] "Finite precision effects can lead to small numerical 


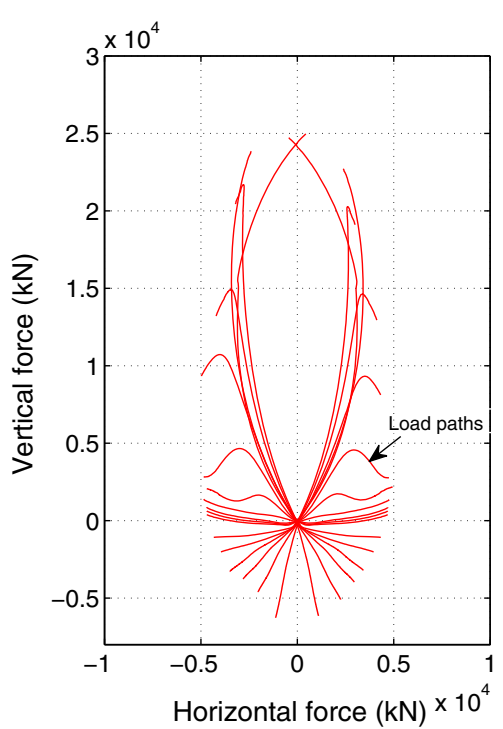

(a)

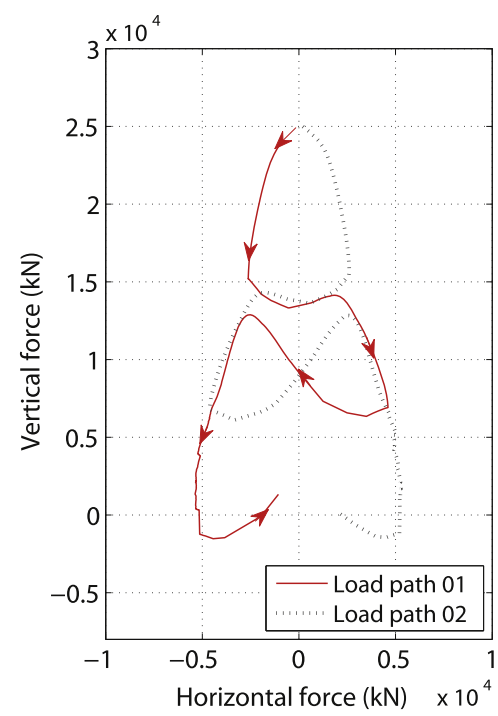

(c)

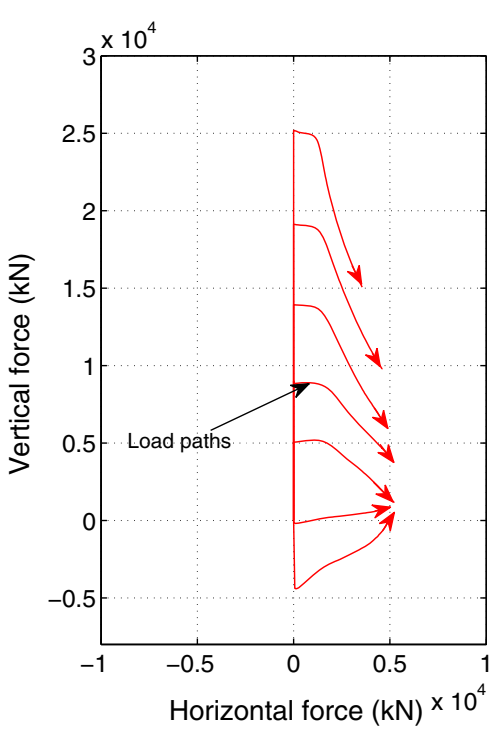

(b)

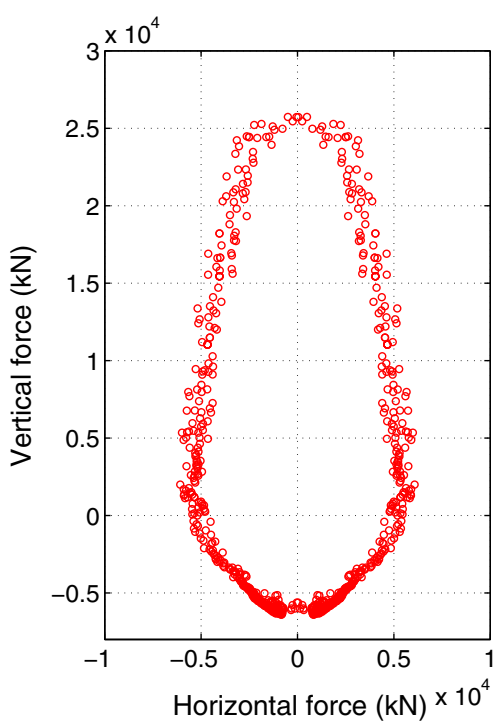

(d)

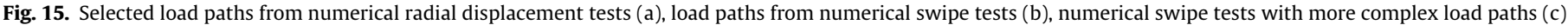
and complete results from numerical radial displacement test (d), in $H-V$ plane $(M=0)$.

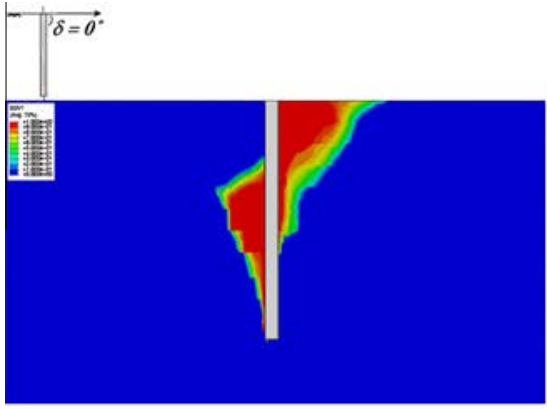

(a)

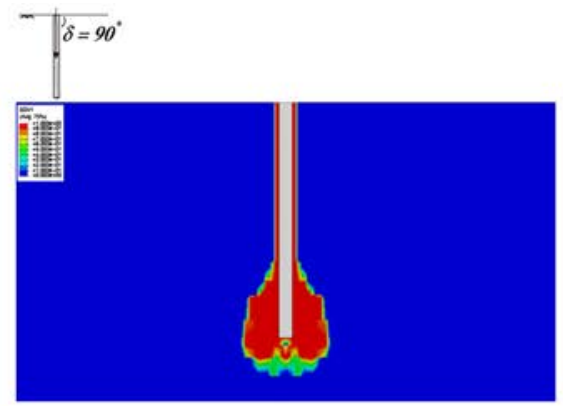

(b)

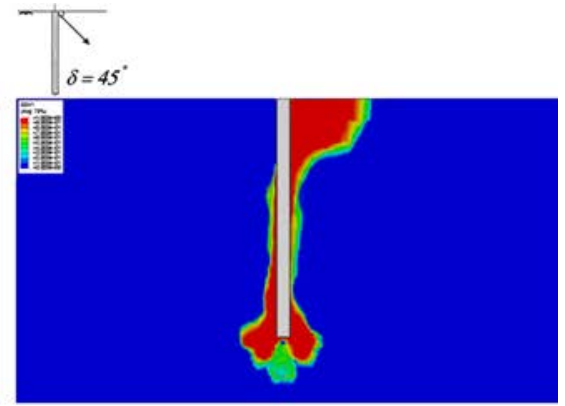

(c)

Fig. 16. Plastic zone under (a) pure horizontal loading $\left(0^{\circ}\right)$ (b) pure vertical loading $\left(90^{\circ}\right)$ and (c) inclined loading $\left(45^{\circ}\right)$. 
differences when running jobs on different numbers of processors". Multiple processors (4 CPUs) were used at first and the results were similar to the ones presented in Fig. 15(d) but with the $H-V$ point cloud slightly not symmetric to the vertical axis. For the case of a single CPU however, results are found symmetric with respect to the vertical axis, see Fig. 15(d).

\subsubsection{Numerical failure envelope in the $H-M$ plane}

The failure envelope is hereafter investigated in the $H-M$ plane and for different vertical loading levels. The procedure is divided in two steps: first, the pile is loaded until a certain vertical force $V_{i}$. Then, radial displacement loadings are applied considering a constant ratio between the combined rotation-displacements increments. The ratio between the increments of rotation $\theta$ and horizontal displacement $u$ is constant $(\delta \theta / \delta u \equiv$ constant), which implies a straight loading path, see Fig. 17.

In analogy with the $H-V$ plane, load paths in the $H-M$ plane start from the origin and stop at the failure envelope, see Fig. 18(a). The main results are summarized hereafter (Figs. 18(b) and 19 for different vertical load levels):

- The failure envelopes present a remarkable inclined elliptical shape.

- The presence of bending moment has a significant influence on the horizontal bearing capacity of the pile. The contribution depends on the loading direction (or the respective signs of the horizontal force and bending moment).

- The influence of the vertical load on the inclination of the bearing capacity diagrams is negligible.

- The increase of the vertical load decreases the size of the bearing capacity diagram (similar trends have been found in compression and in tension).

The shape and inclination of the failure envelope can be explained as follows: the presence of a positive bending moment on the pile head increases the horizontal force bearing capacity, see the area with the arrow 1 in Fig. 18(b). The positive bending moment will restrain the pile deformations caused by a positive horizontal force. To overcome the counterbalance effect of the bending moment, an increased horizontal force is required in order to reach the ultimate state. Therefore, the required horizontal loading is higher than in point A. Part 1 can be seen as the horizontal force dominated failure part.

For the same reason, and in order to overcome the horizontal force, a greater bending moment is needed. The required bending moments are larger than the failure moment at point B. Part 2 can be seen as the bending moment dominated part. If now the sign of the horizontal force and bending moment are opposite, the presence of bending moment accelerates the failure of the pile soil system and the horizontal forces are reduced, Fig. 18(b) part 3.

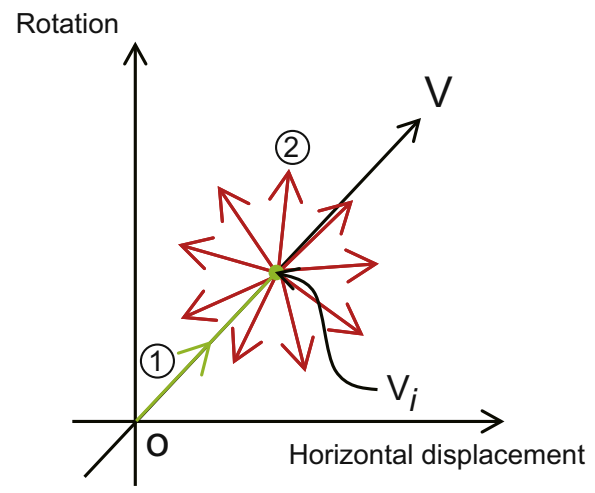

Fig. 17. Radial displacements at the vertical load level $V_{i}$.

\subsubsection{Numerical failure envelope in the $H-M-V 3 D$ space}

By combining the results in the $H-V$ plane (Fig. 15) and in the $H-M$ plane for different vertical load levels (Figs. 18(b) and 19), the complete failure envelope is plotted in Fig. 20. All the numerical data points belong to a 3D surface that has inclined elliptical cross-sections along the vertical force axis.

From the numerical radial tests in Fig. 20 the horizontal bearing capacity $(V=0$ and $M=0)$ is estimated at $H_{0}=5000 \mathrm{kN}$, the vertical compression bearing capacity $(H=0$ and $M=0) V_{c 0}=25,000 \mathrm{kN}$ the vertical tension bearing capacity $(H=0$ and $M=0)$ $V_{t 0}=5100 \mathrm{kN}$ and the ultimate bending resistance $(H=0$ and $V=0) M_{0}=0.42 \times 10^{5} \mathrm{kN} \mathrm{m}$. These values play an important role in the construction of the analytical relationship of the 3D failure envelope for a single vertical pile in sand presented in the following section. One can however notice that the mentioned capacities are greater than those often used in practice. A possible explanation is that the ultimate strength is calculated using a finite element model without any specific safety factors, under the assumption of large deformations and with an advanced re-meshing technique (ALE). Furthermore, the zoom presented in Fig. 14 shows that the ultimate strength is defined as the final the nonconverged point and not on the prior smooth part of the load path.

\subsection{Analytical equations}

\subsubsection{Analytical equation for the failure envelope in the $H-V$ plane}

In the $H-V$ plane there is no bending moment and thus a pinned connection can be considered between the pile head and the pile cap (no moments are transmitted). Meyerhof and Ranjan [2] proposed a semi-empirical formula to evaluate the interaction between the horizontal and vertical forces that reads:

$\left(\frac{H}{H_{0}}\right)^{2}+\left(\frac{V}{V_{0}}\right)^{2}=1$

where $H_{0}$ and $V_{0}$ are the horizontal and vertical bearing capacities of the pile. Eq. (17) can be written in a normalized form as follows:

$f=m^{2}+v^{2}-1$

where $m=H / H_{0}$ and $v=V / V_{c 0}$ in compression or $v=V / V_{t 0}$ in tension. $m$ and $v$ are dimensionless quantities.

The comparison of the semi-empirical Eq. (18) with the numerical results is shown in Fig. 21(a) and (b). The agreement is satisfactory although some discrepancies are identified in the tension part (dash line). A small amplification of the differences is also seen in the normalized curve that is often used in the macro-element technology [16-18]. Nevertheless, the agreement is considered acceptable and Eq. (18) is used hereafter for the tension and compression parts. Therefore, and in order to determine the failure envelope of a single pile in sand in the $H-V$ plane three parameters are necessary: the horizontal bearing capacity $H_{0}$, the vertical compression capacity $V_{c 0}$ and the vertical tension capacity $V_{t 0}$.

A strong interaction between the vertical and the horizontal forces is observed (see Fig. 21(b)) when the vertical load exceeds $50 \%$ of the ultimate compression bearing capacity. This may be due to the size of the soil deposit in the FEM model that is relatively small (especially when considering the distance from the pile end to the bottom boundary). Achmus and Thieken [12] pointed out that with a relatively larger FE model, this interaction may be found reduced.

\subsubsection{Analytical equation for the failure envelope in the $H-M-V 3 D$ space}

As shown in Section 4.2.2, the failure envelope in the $H-M$ plane has an inclined elliptical shape. Inspired from the work of Gottardi et al. [51], where the authors proposed a formula to reproduce an 


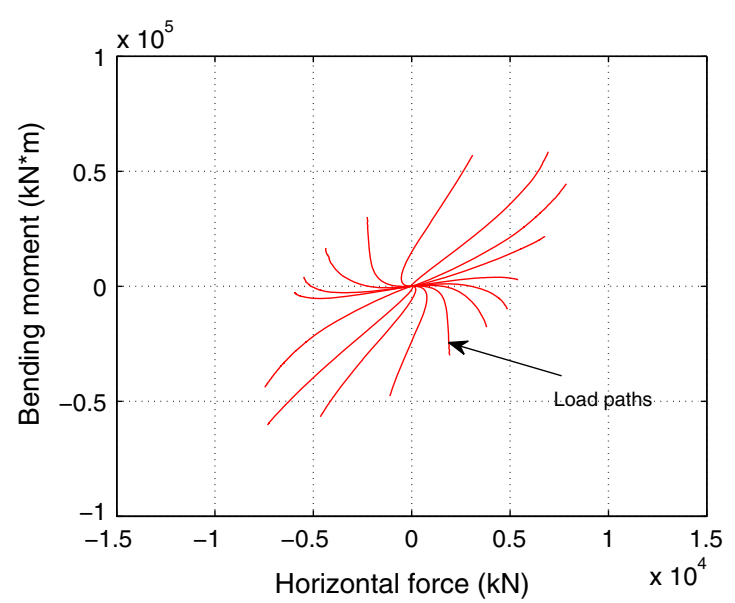

(a)

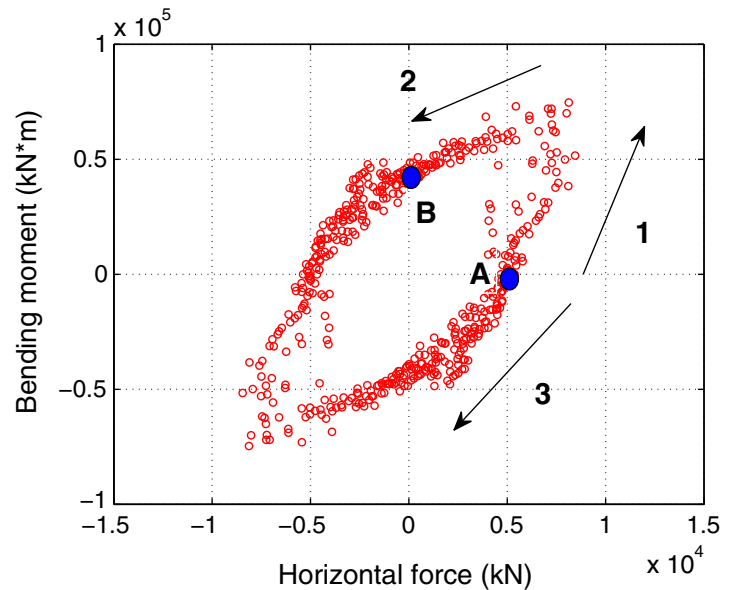

(b)

Fig. 18. (a) Load paths and (b) numerical radial displacement tests in the $H-M$ plane corresponding to $V=0$.

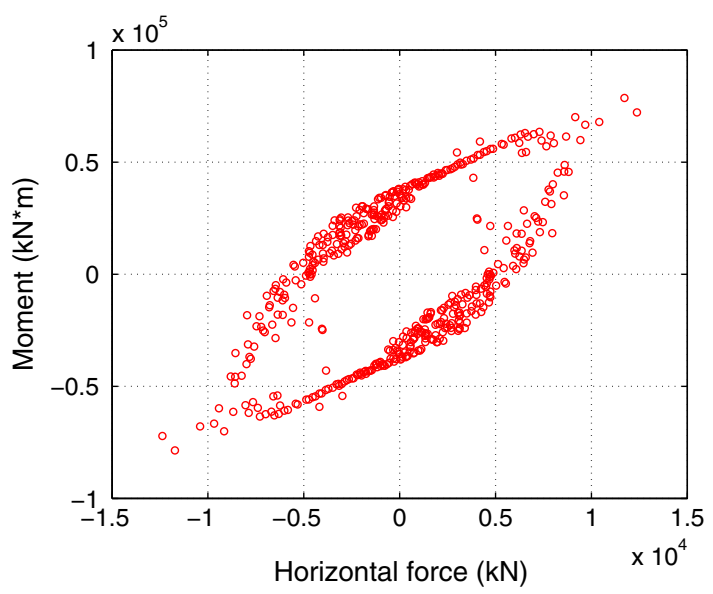

(a)

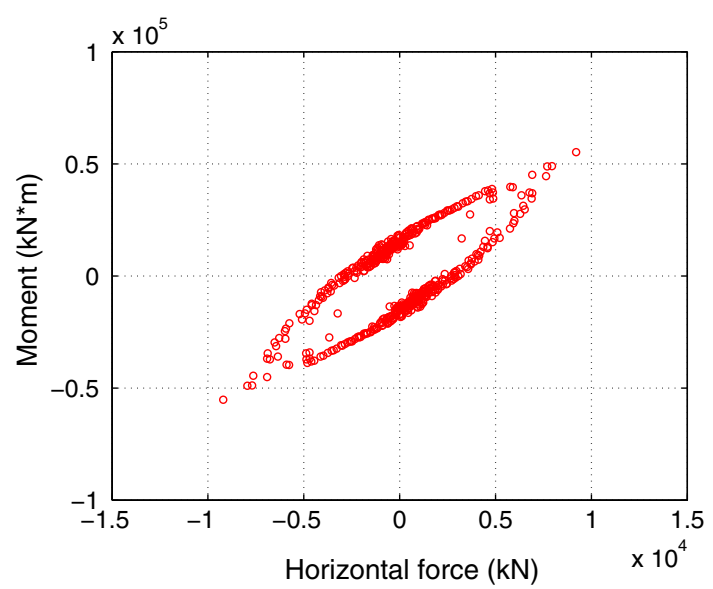

(c)

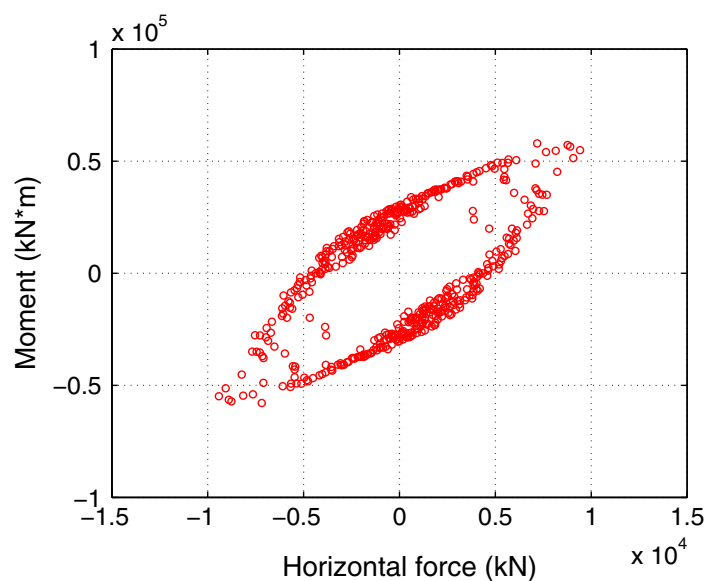

(b)

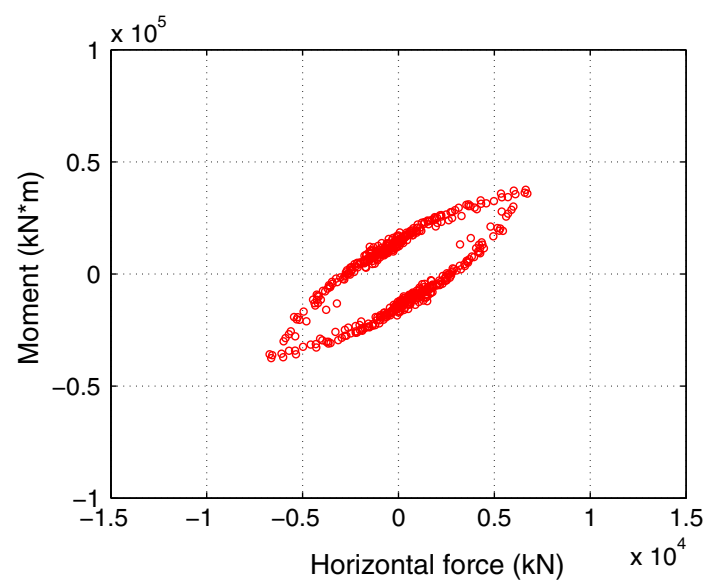

(d)

Fig. 19. Failure envelopes at different vertical load levels in the $H-M$ plane: (a) $V=1.0 \times 10^{4} \mathrm{kN}(\mathrm{b}) V=1.5 \times 10^{4} \mathrm{kN}(\mathrm{c}) V=2.0 \times 10^{4} \mathrm{kN}(\mathrm{d})$ and $V=2.25 \times 10^{4} \mathrm{kN}$.

inclined elliptical failure envelope valid for circular footings, a similar equation is proposed hereafter for a single pile in sand:

$f=\alpha m^{2}+\xi n^{2}-\beta m n-\rho(v)=0$ where $m=H / H_{0}$ the normalized horizontal force, $n=M / M_{0}$ the normalized bending moment and $v=V / V_{c 0}$ or $v=V / V_{t 0}$ the normalized vertical force (dependent on the sign of the vertical load). $\alpha, \xi, \beta$ and $\rho$ are constants that control the shape of the ellipse. 


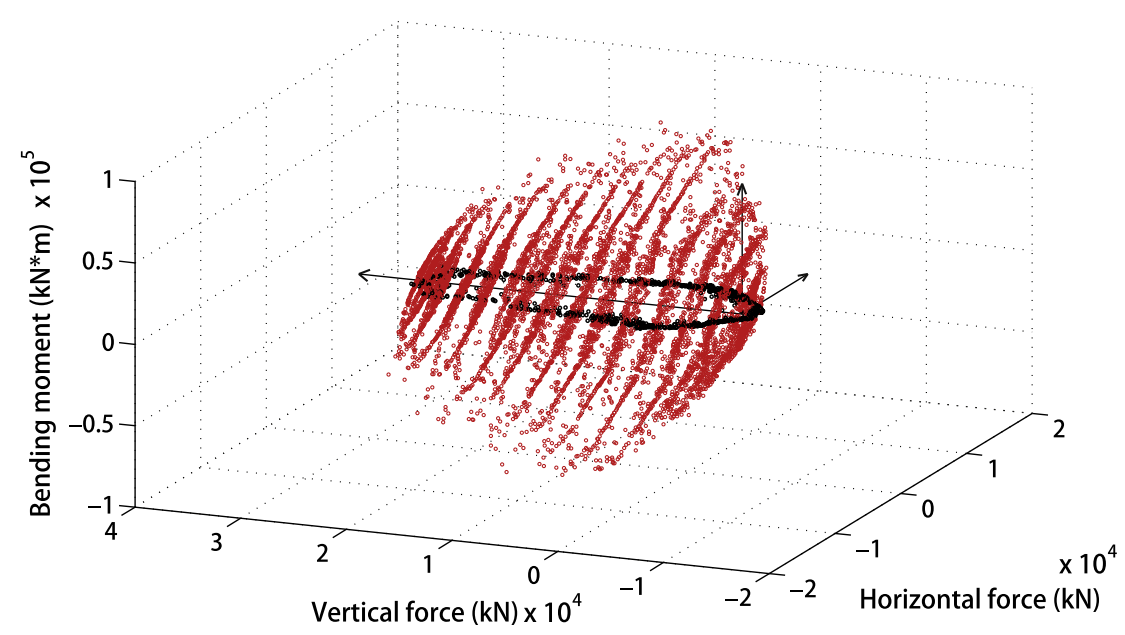

Fig. 20. Numerical failure envelope in the $H-M-V$ 3D space.

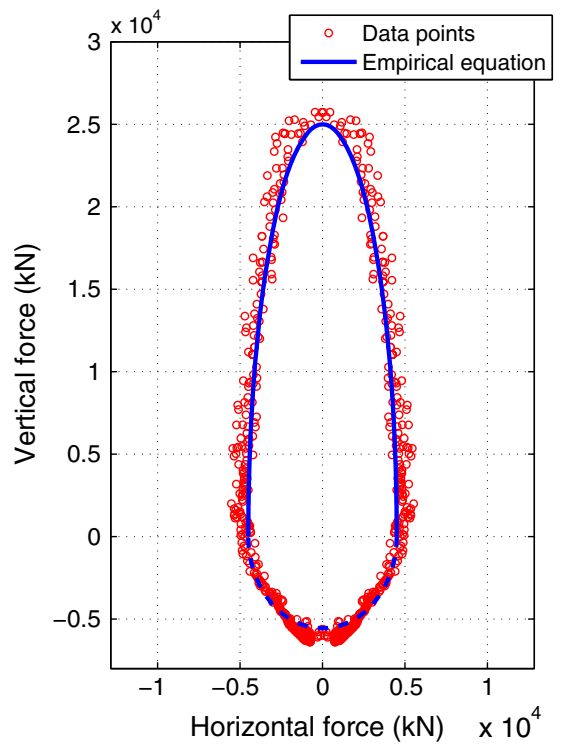

(a)

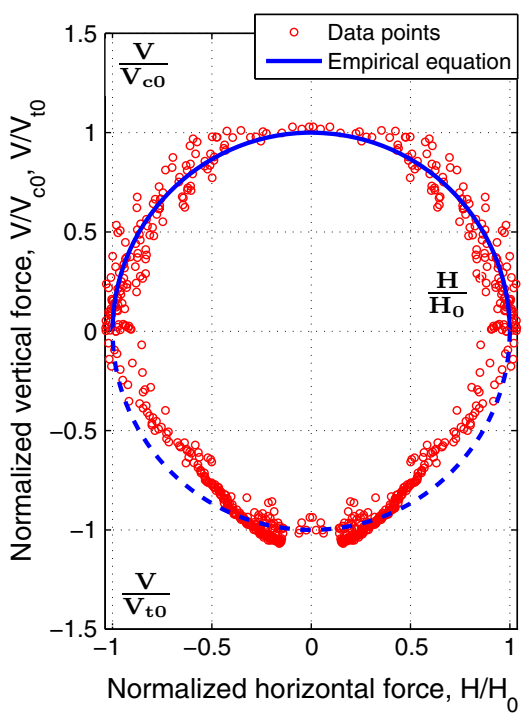

(b)

Fig. 21. Comparison of Eq. (18) with the numerical results (a) in the $H-V$ plane and (b) in the normalized $\frac{H}{H_{0}}-\frac{V}{V_{c 0}}-\frac{V}{V_{t 0}}$ space.

Eq. (19) is an inclined ellipse in the $H-M$ plane where the parameter $\alpha$ controls the intersections of the curve with the $H$ axis, $\beta$ with the $M$ axis and $\rho$ the isotropic expansion or contraction of the ellipse; the combination of $\alpha, \xi$ and $\beta$ controls the inclination of the ellipse.

The parameters are first fitted using the normalized numerical data in the $H-M$ plane at zero vertical force. They are found equal to $\alpha=1.0, \xi=1.0, \beta=1.5$ and $\rho=1.0$, Eq. (19) thus becomes:

$f=1.0 m^{2}+1.0 n^{2}-1.5 m n-1.0=0$

The fitted curves are plotted in Fig. 22(a) and (b) and shows a good agreement with the numerical data both in the $H-M$ and in the normalized $H / H_{0}-M / M_{0}$ plane. As discussed in Section 4.2.2, the vertical load influences the size of the elliptical cross-sections but not their inclinations, see Fig. 19. In order to introduce this behavior, it is proposed hereafter to link the parameter $\rho$ in Eqs. (19) and (20) with the vertical load as follows:

$f=1.0 m^{2}+1.0 n^{2}-1.5 m n-\left(1-v^{2}\right)=0$

Fig. 23 shows the comparison of the numerical results with Eq. (21) in the normalized $H / H_{0}-M / M_{0}$ plane and this at different vertical force levels. Although the proposed equation cannot accurately fit all the data points, especially for high vertical force levels (see for example Fig. 23(c) and (d)), it captures the main features of the behavior. The proposed equation is therefore considered hereafter satisfactory.

When $n=0$ (corresponding to zero moment at the pile head) Eq. (21) becomes equal to Eq. (18). In other words, the proposed equation is applicable for both pinned pile-head conditions in the $H-V$ plane and constrained pile-head conditions in the $H-M-V$ space.

The 3D failure envelope provided by Eq. (21) is plotted from different view points in Fig. 24(a) and (b). Fig. 24(c) contains the 3D failure envelope in the normalized $H / H_{0}, M / M_{0}, V / V_{c 0}$, and $V / V_{t 0}$ space. The tension part of the failure envelope is marked with a dark color.

Finally, the 3D failure envelope for a single vertical pile in sand defined by Eq. (21) is plotted in Fig. 25 together with all the numerical data points. The proposed surface reproduces the 3D distribution of the numerical data with a correlation factor $R^{2}$ estimated around 0.89 . Key parameters for the 3D failure envelope are: the horizontal bearing capacity $H_{0}$, the vertical compression capacity $V_{c 0}$, the vertical tension capacity $V_{t 0}$ and the bending capacity $M_{0}$. 


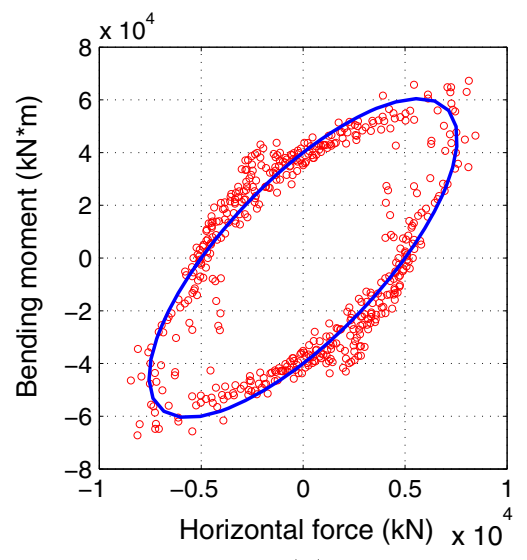

(a)

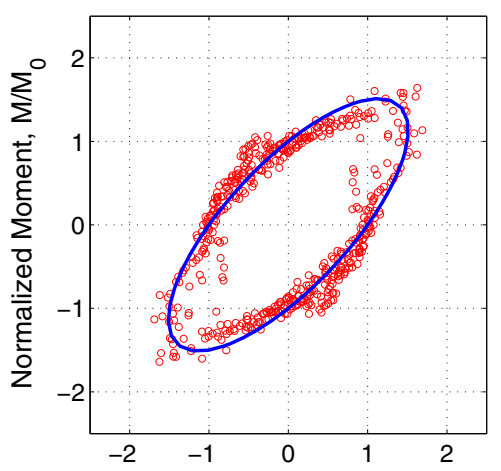

Normalized Horizontal force, $\mathrm{H} / \mathrm{H}_{0}$

(b)

Fig. 22. Comparison of Eq. (20) with the numerical results (a) in the $H-M$ plane and (b) in the normalized $\frac{H}{H_{0}}-\frac{M}{M_{0}}$ plane.

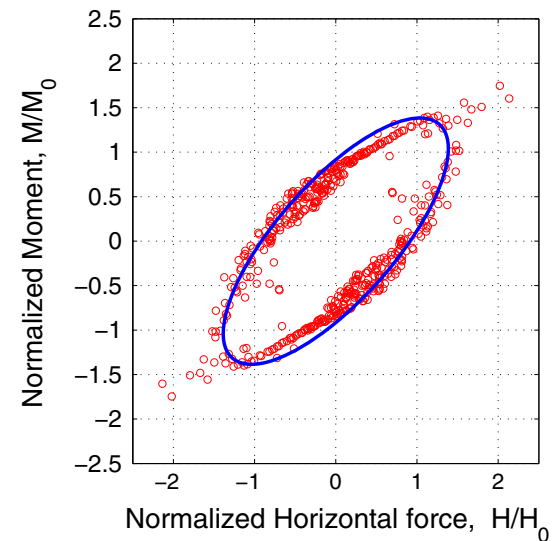

(a)

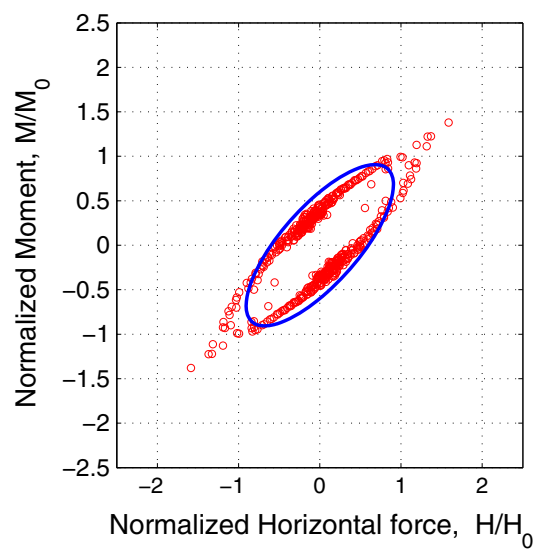

(c)

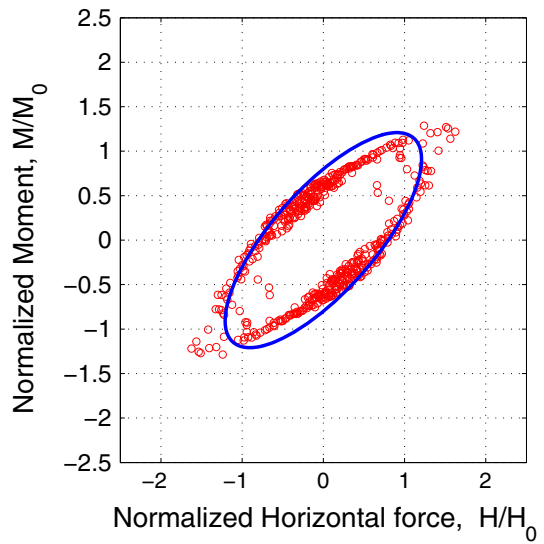

(b)

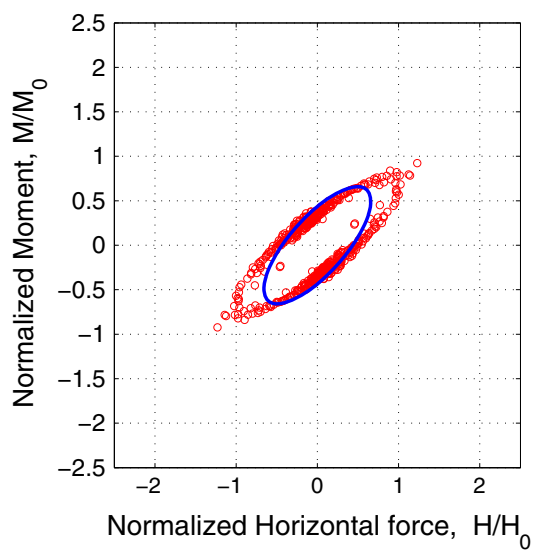

(d)

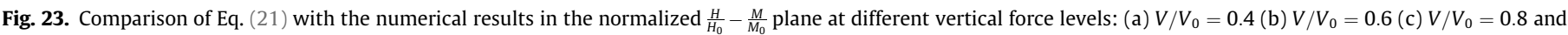
(d) $V / V_{0}=0.9$

\section{Limitations of the approach}

The study of the failure envelope in the $H-M-V$ space is based on the assumption that the pile stays elastic. However, plasticity of the pile should be sometimes considered in engineering design. A simple way to take this into account is to consider that the pile has a limited bending capacity $M_{y}$ that the maximum bending resistance of the soil-pile system cannot exceed. Therefore, the original failure envelope valid for an elastic pile, Fig. 26(a), could be modified assuming that the plastic bending response of the pile neither hardens nor softens, as indicated (in blue in Fig. 26(b)). Nevertheless, extensive finite elements calculations are necessary in order to validate this new shape.

The soil constitutive law adopted in the paper does not take into account the influence of the third stress invariant (Lode angle independent) and thus the shearing strength in extension is 


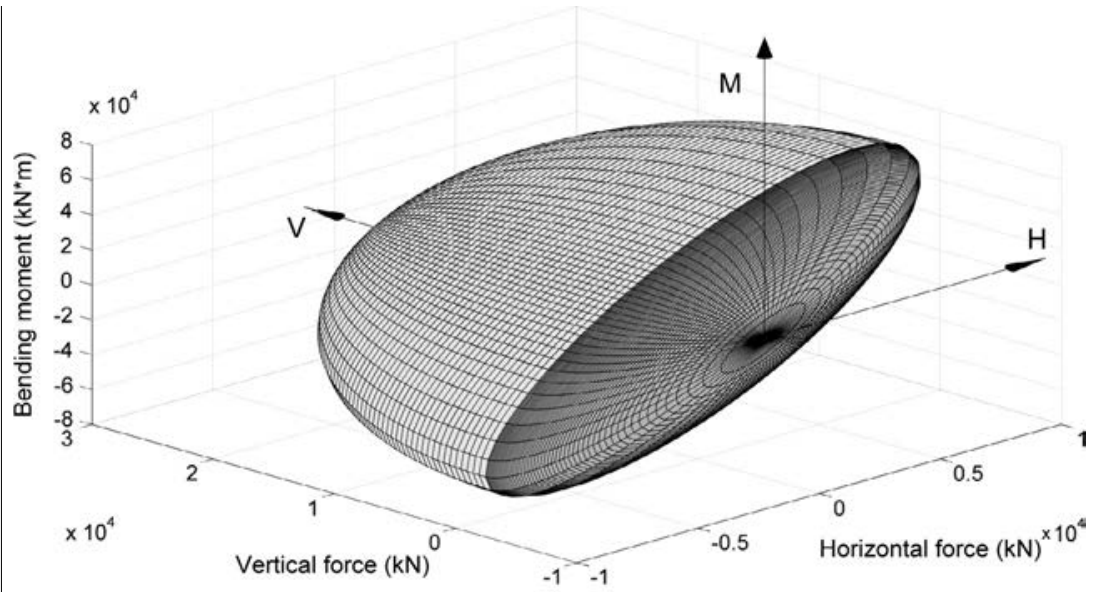

(a)

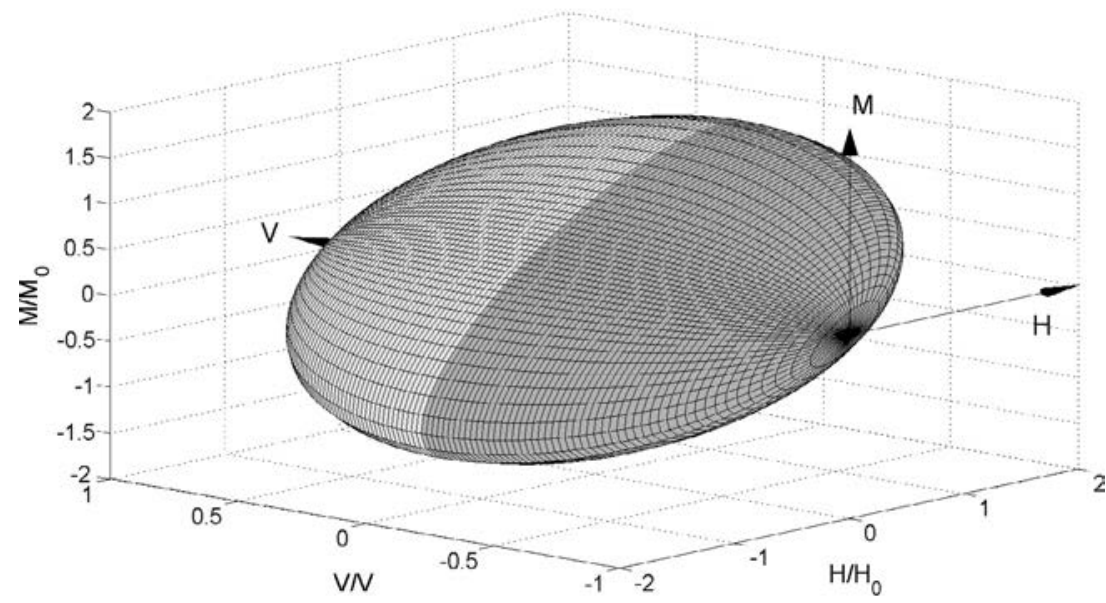

(b)

Fig. 24. 3D failure envelope provided by Eq. (21): (a) in 3D space and (b) in the normalized $\frac{H}{H_{0}}-\frac{M}{M_{0}}-\frac{V}{V_{c 0}}-\frac{V}{V_{t 0}}$ space.

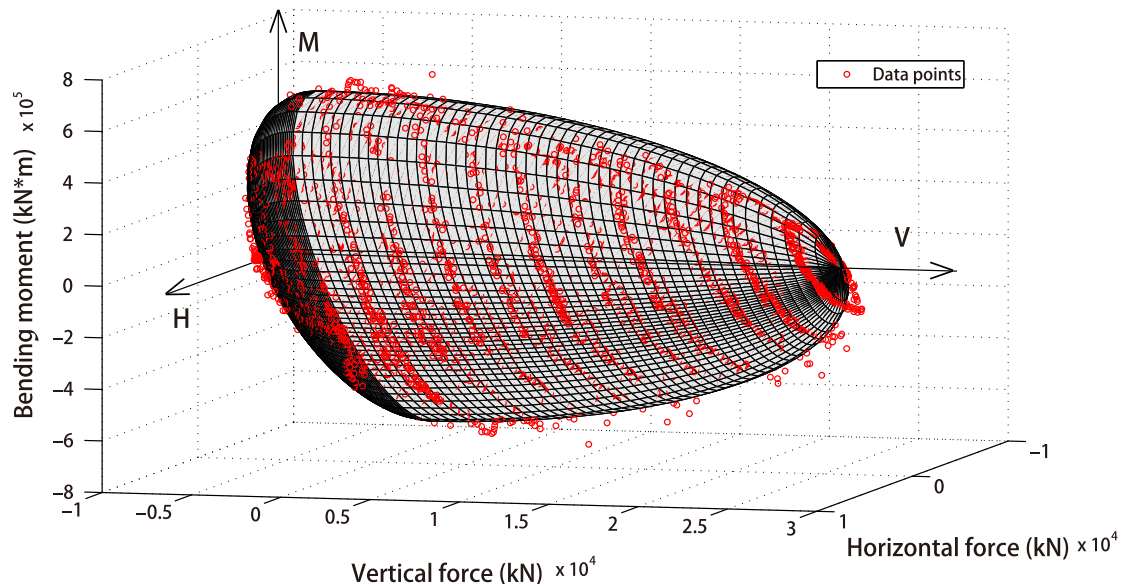

Fig. 25. 3D failure envelope for a single vertical pile in sand: yield surface provided by Eq. (21) vs. numerical data points.

overestimated. Furthermore, the critical state cannot be captured. More advanced constitutive soil model should be considered in the future to extend the validity of the approach.
Finally, the distance from the pile tip to the bottom of the finite element model can influence the failure envelope. This aspect should also be addressed in future studies. 


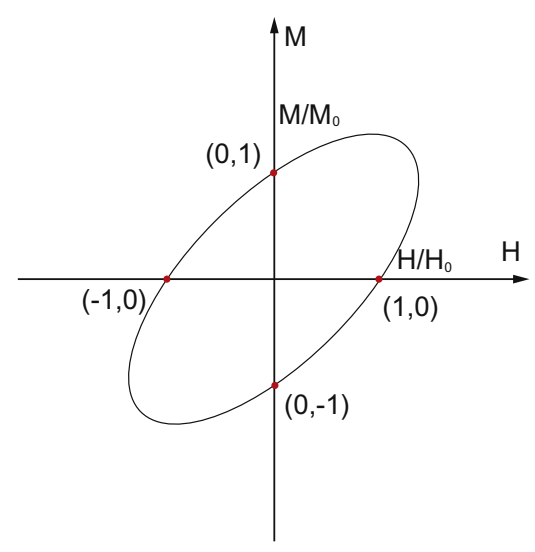

(a)

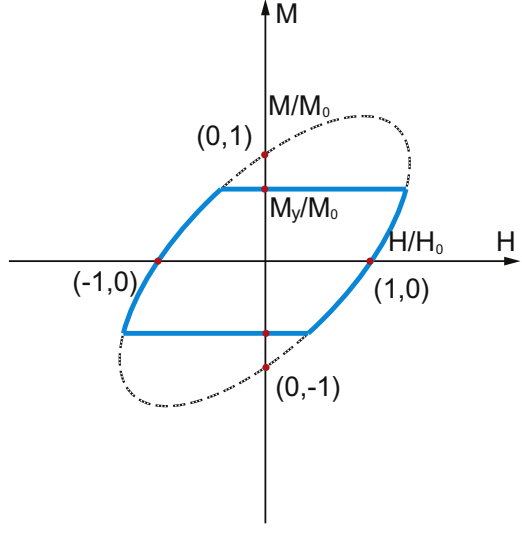

(b)

Fig. 26. Schematic of (a) yield surface for elastic pile $(V=0)$ and (b) yield surface after considering pile plasticity $(V=0)$.

\section{Conclusions}

This paper presents a comprehensive numerical study of the 3D failure envelope of a single elastic pile in sand. A simple hypoplastic constitutive law is adopted for the nonlinear behavior of the sand and an important number of numerical tests are carried out mainly using the radial displacement method. The 3D interaction diagram of the vertical force, horizontal force, and bending moment is investigated.

It is found that the vertical load significantly influences the horizontal bearing capacity and the ultimate bending moment while the failure envelope in the $H-M$ plane presents an inclined elliptical shape. Analytical equations are proposed to reproduce the 3D failure diagram of a single pile in sand valid either for a pinned connection in the $H-V$ plane or a moment connection with the pile cap or the super-structure in the $H-V-M$ space. The key parameters for the analytical equations are relatively easy to determine either experimentally or numerically making the above equations useful for engineering design offices. The work presented in this paper focuses on the failure envelope of a flexible pile in sand and needs to be generalized in the future to different soils and pile stiffness.

\section{Acknowledgments}

The financial support of IFSTTAR (Institut français des sciences et technologies des transports, de l'aménagement et des réseaux) and of the Région Pays de la Loire is gratefully acknowledged. The authors would like also to thank the valuable support and help of the technical staff of the IFSTTAR centrifuge team. Dr. Matthieu Blanc and Dr. Luc Thorel from IFSTTAR provided the experimental data from the national french programme 'Projet National SOLCYP' which is partially funded by ANR (l'Agence Nationale de la Recherche) and IREX (L'Institut pour la Recherche appliquée et l'Expérimentation en Génie Civil). They are also hereafter greatly acknowledged.

\section{References}

[1] Meyerhof GG. The design of Franki piles with special reference to group in sand. In: Proceedings symposium on pile foundation. Stockholm, Sweden; 1960. p. $105-23$.

[2] Meyerhof GG, Ranjan G. The bearing capacity of rigid piles under inclined loads in sand. I: Vertical piles. Can Geotech J 1972;9(4):430-46.

[3] Ranjan G. The bearing capacity of batter piles under inclined loads in sand Ph.D. thesis. Nova Scotia Technical College; 1970.

[4] Meyerhof GG, Ranjan G. The bearing capacity of rigid piles under inclined loads in sand. II: Batter piles. Can Geotech J 1973;10(1):71-85.
[5] Meyerhof GG, Ranjan G. The bearing capacity of rigid piles under inclined loads in sand. III: pile groups. Can Geotech J 1973;10(3):428-38.

[6] Meyerhof GG, Yalcin AS. Behaviour of flexible batter piles under inclined loads in layered soil. Can Geotech J 1993:30(2):247-56.

[7] Sastry VVRN, Meyerhof GG. Behaviour of flexible piles in layered sands under eccentric and inclined loads. Can Geotech J 1994;31(4):513-20.

[8] Meyerhof GG, Yalcin AS. Bearing capacity of flexible batter piles under eccentric and inclined loads in layered soil. Can Geotech J 1994:31(4):583-90.

[9] Das BM, Seeley GR, Raghu D. Uplift capacity of model piles under oblique loads. J Geotech Geoenviron Eng 1976;102(9):1009-13.

[10] Patra NR, Pise PJ. Model pile groups under oblique pullout loads-an investigation. Geotech Geol Eng 2006;24(2):265-82.

[11] Cho Y. Inclined loading capacity of suction piles. In: Proceedings of the twelfth international offshore and polar engineering conference. The international society of offshore and polar engineers, vol. 3. Kitakyushu, Japan; 2002. p. 82732 .

[12] Achmus M, Thieken K. On the behavior of piles in non-cohesive soil under combined horizontal and vertical loading. Acta Geotechnica 2010;5(3):199-210. http://dx.doi.org/10.1007/s11440-010-0124-1.

[13] Mroueh H, Shahrour I. Numerical analysis of the response of battered piles to inclined pullout loads. Int $\mathrm{J}$ Numer Anal Methods Geomech 2009;33(10):1277-88. http://dx.doi.org/10.1002/nag.

[14] Fan Q, Meng X. Failure envelope of pipe pile foundation under combined loading. In: Advances in pile foundations, geosynthetics, geoinvestigations, and foundation failure analysis and repairs; 2011. p. 109-16. doi:10.1061/ $47631(410) 13$.

[15] Correia AA. A pile-head macro-element approach to seismic design of monoshaft-supported bridges, Ph.D. thesis. Università degli Studi di Pavia \& Istituto Universitario di Studi Superiori; 2011.

[16] Nova R, Montrasio L. Settlements of shallow foundations on sand. Géotechnique 1991;41(2):243-56.

[17] Grange S, Kotronis P, Mazars J. A macro-element to simulate dynamic soilstructure interaction. Eng Struct 2009;31(12):3034-46. http://dx.doi.org/ 10.1016/i.ijsolstr.2009.06.015.

[18] Grange S, Kotronis P, Mazars J. A macro-element to simulate 3D soil-structure interaction considering plasticity and uplift. Int J Solids Struct 2009;46(20):3651-63. http://dx.doi.org/10.1016/i.ijsolstr.2009.06.015.

[19] Grange S, Botrugno L, Kotronis P, Tamagnini C. The effects of soil-structure interaction on a reinforced concrete viaduct. Earthquake Eng Struct Dyn 2011;40(1):93-105. http://dx.doi.org/10.1002/eqe.1034.

[20] Mohr O. Welche Umstände bedingen die Elastizitätsgrenze und den Bruch eines Materials? Zeitschrift des Vereins Deutscher Ingenieure 1900;46:1524-30.

[21] Drucker D, Prager W. Soil mechanics and plastic analysis or limit design. Q Appl Math 1952;10(2):157-65.

[22] Dafalias YF. Bounding surface plasticity. I: mathematical foundation and hypoplasticity. J Eng Mech 1986;112(9):966-87.

[23] Bardet J. Hypoplastic model for sands. J Eng Mech 1990;116(9):1973-94.

[24] Kolymbas D. An outline of hypoplasticity. Arch Appl Mech 1991;61(3):143-51.

[25] von Wolffersdorff PA. A hypoplastic relation for granular materials with a predefined limit state surface. Mech Cohes-Frict Mater 1996;1(3):251-71.

[26] Niemunis A. Extended hypoplastic models Dissertation submitted for habilitation, Habilitation thesis. Ruhr-University, Bochum; 2002.

[27] Darve F. Une formulation incrémentale des lois rhéologiques. Application aux sols, Ph.D. thesis. INP Grenoble; 1978.

[28] Tamagnini C, Viggiani G, Chambon R. A review of two different approaches to hypoplasticity. In: Constitutive modelling of granular materials. Berlin Heidelberg: Springer; 2000. p. 107-45. http://dx.doi.org/10.1007/978-3-64257018-6 5 . 
[29] Drnevich BO, Hardin VP. Shear modulus and damping in soils: design equations and curves. J Soil Mech Found Div 1972;98:667-92.

[30] Iwasaki T, Tatsuoka F. Effects of grain size and grading on dynamic shear moduli of sands. Soils Found 1977;17(3):19-35.

[31] Iwasaki T, Tatsuoka F, Takagi Y. Shear modulus of sands under tosional shear loading. Soils Found 1978;18(1):39-56.

[32] Ishihara K. Soil behaviour in earthquake geotechnics. Oxford: Clarendon Press; 1996.

[33] Yu HS. Plasticity and geotechnics. Springer; 2006.

[34] Borja R. Plasticity: modeling \& computation. Springer; 2013.

[35] Dafalias Y, Herrmann L. Bounding surface formulation of plasticity. John Wiley and Sons, Ltd.; 1982.

[36] Bardet JP. Bounding surface plasticity model for sands. J Eng Mech 1986;112(11):1198-217.

[37] Andria-Ntoanina I, Canou J, Dupla JC. Caractérisation mécanique du sable de Fontainebleau NE34 à lappareil triaxial sous cisaillement monotone, Tech. rep., Laboratoire Navier - Géotechnique (CERMES, ENPC/LCPC); 2010.

[38] Delfosse-Ribay E, Djeran-Maigre I, Cabrillac R, Gouvenot D. Shear modulus and damping ratio of grouted sand. Soil Dyn Earthquake Eng 2004;24(6):461-71. http://dx.doi.org/10.1016/i.soildyn.2004.02.004.

[39] Li Z, Escoffier S, Kotronis P. Using centrifuge tests data to identify the dynamic soil properties: application to Fontainebleau sand. Soil Dyn Earthquake Eng 2013;52:77-87. http://dx.doi.org/10.1016/i.soildyn.2013.05.004.

[40] Schofield A, Zeng X. Design and performance of an equivalent-shear-beam container for earthquake centrifuge modelling. Géotechnique 1996;46(1):83-102.

[41] Teymur B, Madabhushi SPG. Experimental study of boundary effects in dynamic centrifuge modelling. Géotechnique 2003;53(7):655-63.

[42] Zeghal M, Elgamal A. Analysis of site liquefaction using earthquake records. J Geotech Eng 1994;120(6):996-1017. http://dx.doi.org/10.1061/. ASCE)07339410(1994)120:6(996.

[43] Giannakos S, Gerolymos N, Gazetas G. Cyclic lateral response of piles in dry sand: Finite element modeling and validation. Comput Geotech 2012;44:116-31.

[44] Abaqus/Standard, Abaqus 6.10 Documentation (SIMULIA Abaqus 6.10), Dassault Systèmes, 2010.

[45] Rosquoët F. Pile under lateral cyclic load, Ph.d. thesis. Ecole Centrale de Nantes \& Université de Nantes; 2004.

[46] Guefrech A, Rault G, Chenaf N, Thorel L, Garnier J, Puech A. Stability of cast in place piles in sand under axial cyclic loading. In: Proceeding of the 7th international conference on offshore site investigation and geotechnics. London, UK: Royal Geographical Society; 2012. p. 239-334.
[47] Poulos HG, Davis EH. Pile foundation analysis and design. Inc., New York: John Wiley and Sons; 1980.

[48] Jardine RJ, Potts DM, Fourie AB, Burland JB. Studies of the influence of nonlinear stress-strain characteristics in soil-structure interaction. Géotechnique 1986;36(3):377-96. http://dx.doi.org/10.1680/geot.1986.36.3.377.

[49] Mair RJ. Developments in geotechnical engineering research: applications to tunnels and deep excavations. Unwin Memorial Lecture 1992. In: Proc. Instn Civ. Engrs Civ. Engng; 1993. p. 27-41.

[50] Santos JA, Correia AG. Reference threshold shear strain of soil. Its application to obtain an unique strain-dependent shear modulus curve for soil. In: Proceedings of the fifteenth international conference on soil mechanics and geotechnical engineering. Istanbul, Turkey; 2001. p. 267-70.

[51] Gottardi G, Houlsby G, Butterfield R. Plastic response of circular footings on sand under general planar loading. Géotechnique 1999;49(4):453-69.

[52] Tan FS. Centrifuge and theoretical modelling of conical footings on sand, Ph.D thesis. University of Cambridge; 1990.

[53] Martin CM, Houlsby GT. Combined loading of spudcan foundations on clay: laboratory tests. Geotéchnique 2000;50(4):325-38.

[54] Bransby MF, Randolph MF. Combined loading of skirted foundations. Geotéchnique 1998;48(5):637-55.

[55] Gerolymos N, Zafeirakos A, Souliotis C. Insight to failure mechanisms of caisson foundations under combined loading: a macro-element approach. In: Proc. 2nd int. conf. on performance-based design in earthquake geotechnical engineering. Taormina, Italy; 2012.

[56] Calvetti F, Prisco C, Nova R. Experimental and numerical analysis of soil-pipe interaction. J Geotech Geoenviron Eng 2004;130(12):1292-9.

[57] Butler HD, Hoy HE. Users manual for the Texas quick-load method for foundation load testing, Tech. rep., Federal Highway Administration, Office of Development, Washignton; 1977.

[58] Davisson MT. High capacity piles. In: Lecture series, innovation in foundation construction. ASCE, Illinois Section; 1972. p. 52.

[59] Brinch Hansen J. Discussion of hyperbolic stress-strain response: cohesive soils. J Soil Mech Found Div 1963;89(4):241-2.

[60] Chin KF. Estimation of the ultimate load of piles not carried to failure. In: Proceedings of 2 nd southease asian conference on soil engineering; 1970. p. 81-90.

[61] Verdure L, Garnier J, Levacher D. Lateral cyclic loading of single piles in sand Int J Phys Modell Geotech 2003;3(3):17-28. http://dx.doi.org/10.1680/ ijpmg.2003.3.3.17.

[62] Rosquoët F, Thorel L, Garnier J, Canepa Y. Lateral cyclic loading of sandinstalled piles. Soils Found 2007;47(5):821-32. 